\title{
Chromaticity of the lattice and beam stability in energy recovery linacs
}

\author{
Vladimir N. Litvinenko* \\ Brookhaven National Laboratory, Upton, New York 11973, USA, \\ and Department of Physics and Astronomy, Stony Brook University, Stony Brook, New York 11974, USA
}

(Received 23 December 2011; published 5 July 2012)

\begin{abstract}
Energy recovery linacs (ERLs) are an emerging generation of accelerators that promises to revolutionize the fields of high-energy physics and photon sciences. These accelerators combine the advantages of linear accelerators with that of storage rings, and augur the delivery of electron beams of unprecedented power and quality. The use of superconducting radio-frequency cavities converts ERLs into nearly perfect "perpetuum mobile" accelerators, wherein the beam is accelerated to the desired energy, used, and then yields the energy back to the rf field. However, one potential weakness of these devices is transverse beam breakup instability that could severely limit the available beam current. In this paper, I propose a novel method of suppressing these dangerous effects via a natural phenomenon in the accelerators, viz., the chromaticity of the transverse motion.
\end{abstract}

DOI: 10.1103/PhysRevSTAB.15.074401

PACS numbers: 29.27.- a, 29.20.Ej

\section{INTRODUCTION}

Energy recovery linacs (ERLs, see Fig. 1) belong to a family of recirculating linacs (RLs) that accelerate a beam of charged particles multiple times in the same linear accelerator, accumulating the beam's energy on each pass. The difference between an RL and an ERL is that the latter does not dispose of the accelerated beam; instead its energy is recovered by decelerating the beam to the injection energy (Fig. 1).

In the ERL, low-energy particles are injected and accelerated on the crest (or near it) in the superconducting radiofrequency (SRF) linac to the top energy. After their usage at top energy, the beam's rf phase is shifted by 180 degrees, and the beam is decelerated in the same linac. The latter assures that the beam's energy is recovered into that of the electromagnetic (EM) field in the linac. Finally, the beam is ejected and disposed of at a very low energy compared with the top energy.

In 1965, Tigner suggested the idea of an energy recovery linac [1]. It took some 35 years of development of superconducting radio-frequency (SRF) ERLs to reach beam currents $\sim 10 \mathrm{~mA}$, and energy of few hundreds $\mathrm{MeV}$. While this sufficed for the first spectacular demonstration of generating very high powerful coherent-radiation in $2000[2,3]$ at the Thomas Jefferson Accelerator Facility (JLab), there now is a well-established need for ERLs with $\mathrm{GeV}$-scale energy, and ampere-scale currents for fundamental and applied sciences. Such ERLs would find unique

\footnotetext{
*Corresponding author. vl@bnl.gov
}

Published by the American Physical Society under the terms of the Creative Commons Attribution 3.0 License. Further distribution of this work must maintain attribution to the author(s) and the published article's title, journal citation, and DOI. applications for next-generation light sources [4,5], and similarly for high-energy physics colliders [6-8].

One main challenge toward meeting these goals is the transverse beam breakup instability (TBBU) that especially is severe for SRF recirculating linacs. Early experiments with recirculating SRF accelerators at Stanford [9] and Illinois [10], where this instability occurred at a few microamperes of the average beam current, highlighted this problem. Dipole high-order modes (HOMs) of the SRF cavities were identified as the culprits driving this instability [11,12], and several remedies were developed for raising this threshold [13].

Detailed theoretical approaches and TBBU simulation programs were developed in the late 1980s [14-16]. The renewed interest also stimulated refinements of the TBBU theory, simulation programs [17-19], and their experimental verification [20], all driven by the need for high-current ERLs, and also by the rewards of resolving this complex problem. Nevertheless, strong damping of HOMs in SRF linacs while maintaining high accelerating gradients remains one of the major unsolved issues.

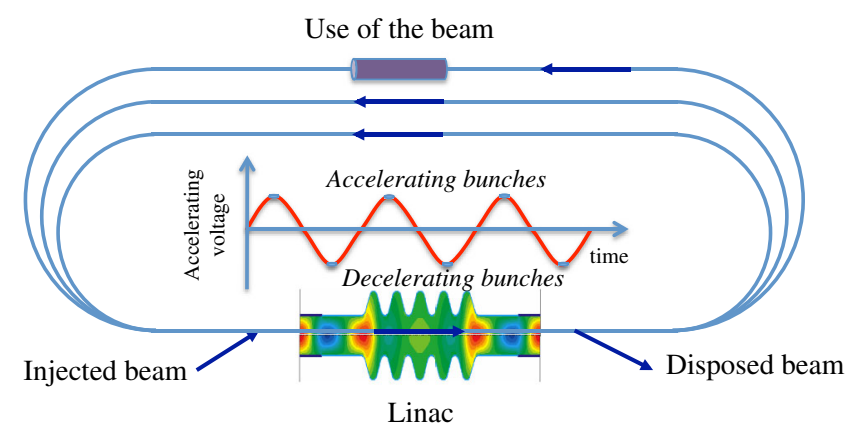

FIG. 1. A simplified sketch of a three-pass ERL comprised of the linear accelerator and three returning loops. 


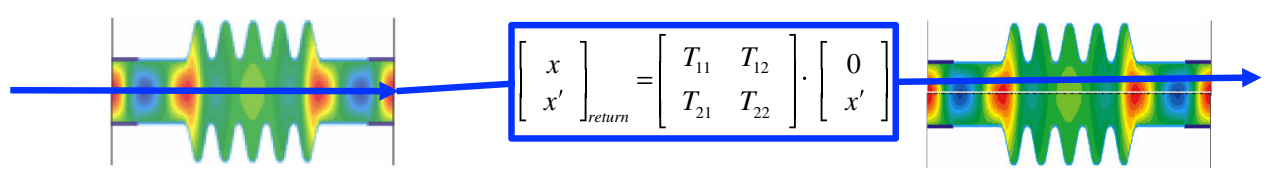

FIG. 2. A sketch of the driving mechanism of TBBU instability in ERLs.

In this paper, I suggest a novel method of addressing TBBU instability, that is, using a natural phenomenon, i.e., the chromaticity ${ }^{1}$ of the ERL's return loops.

Before discussing the working of my proposed suppression mechanism, I reiterate the fundamentals of TBBU instability, sketched in Fig. 2.

Let us consider the SRF cavity with residual oscillations of a dipole transverse HOM that imparts a transverse kick to the passing bunch (indicated by $x^{\prime}$ in Fig. 2). When the bunch returns to the entrance of the same cavity, it acquires a transverse displacement that is proportional to the $T_{12}$ element of the transport matrix (map) of the returning loop

$$
x_{r}=T_{12} \cdot x^{\prime}
$$

which excites the field of the HOM. ${ }^{2}$ The strength of the HOM's longitudinal electric field is proportional to its distance from the axis. For some of the many HOMs, the phasing can be such that the bunch sees a decelerating electric field and loses part of its energy, which is transferred into that of the HOM field. The next bunch in the beam receives a stronger kick, comes back with a larger transverse displacement, and further excites the cavity. Hence, unless the HOM field decays, such a system is prone to instability. The SRF cavities support an environment wherein the $Q$ factor can reach values of $\sim 10^{8}-10^{9}$, i.e., the decay times of the HOMs can be measured in seconds; thus, it is a very challenging problem to reduce the HOMs' $Q$ factors to the required values, $Q \sim 10^{3}$ [21].

In the general ERL case, we can write a complete matrix of dispersion relations [14-19], but an analytical solution of the TBBU's instability threshold can be derived only for a single HOM mode, and a single-pass ERL, and as detailed in [12]:

$$
I_{\mathrm{th}}=\frac{2 c^{2}}{e R_{g} \cdot Q \cdot \omega} \cdot \frac{1}{T_{12} \sin \omega t_{r}},
$$

\footnotetext{
${ }^{1}$ Chromaticity is a natural phenomenon caused by the energy dependence of the focusing strength of the magnetic elements in the ERL's loops.

${ }^{2}$ I focus here on the simple case of uncoupled motion in the loop; hence, the suppression mechanism does not require transverse coupling. As shown in [19], for a dipole HOM polarized at an arbitrary angle, $\theta$, and arbitrary transverse coupling described by a $4 \times 4$ transport matrix $T, T_{12}$ should be replaced in the TBBU dispersion relation by $T_{12} \cos ^{2} \theta+\left(T_{14}+T_{32}\right) \times$ $\sin \theta \cos \theta+T_{34} \sin ^{2} \theta$. Since all considerations for $T_{12}$ are applicable to $T_{12}, T_{14}, T_{32}, T_{34}$, the suppression of the beam response by chromaticity will remain intact.
}

where $c$ is the speed of the light, $e$ is the elementary charge, $R_{g} \cdot Q$ is the HOM's impedance (measured in $\Omega$ ), ${ }^{3} \omega$ is its frequency, and $t_{r}$ is the beam's travel time through the returning loop. Thus, for $T_{12} \sin \omega t_{r}>0$, the $I_{\text {th }}>0$ and TBBU instability occurs for beam current above the threshold, i.e., $I>I_{\mathrm{th}}$. What is important is that, for a given cavity with $2 c^{2} / e R_{g} \omega=$ const, the threshold current is inversely proportional to $Q \cdot\left|T_{12} \sin \omega t_{r}\right|$.

Since there are many HOM modes in the cavities encompassing a large range of the spectrum, there can be modes with $\left|\sin \omega t_{r}\right| \sim 1$; then, the only meaningful way of increasing the threshold is by reducing the values of $Q$ and the $\left|T_{12}\right|$.

As shown in [17], Eq. (66), in an ERL with $N$ passes through its linac, we can estimate the TBBU threshold by

$$
I_{\mathrm{th}}=\frac{2 c^{2}}{e R_{g} \cdot Q \cdot \omega} \cdot \frac{1}{\sum_{J=1}^{2 N} \sum_{I=J+1}^{2 N} T^{I J} \sin \omega\left(t_{I}-t_{J}\right)},
$$

where $T^{I J} \equiv T_{12}\left(s_{J} \mid s_{I}\right)$ is the element of transport matrix between the $J$ th and $I$ th pass through the linac. Analyzing Eq. (3) the authors of Ref. [17] offered their natural conjecture that the TBBU threshold scales as follows:

$$
I_{\mathrm{th}} \propto\left(R_{g} \cdot Q \cdot N^{2}\left\langle\left|T^{I J}\right|\right\rangle\right)^{-1} .
$$

Reasoning that the linac's HOM impedance scales with its length and therefore, for a fixed top energy of the ERL, the geometrical impedance then scales as $R_{g} \sim E_{\text {top }} / N$, and

$$
I_{\mathrm{th}} \propto\left(\tilde{R}_{g} \cdot Q \cdot N\left\langle\left|T^{I J}\right|\right\rangle\right)^{-1},
$$

where $\tilde{R}_{g}$ is HOM impedance for a unit length of the linac. On the other hand, in the linac the typical values of the $\beta$ functions, and therefore the typical values of $T^{I J}$, are proportional to its length, which scales as $\sim 1 / N$ (see footnote 7). This observation may challenge the $N^{2}$ dependence of the TBBU threshold for a properly matched ERL lattice. However, the tendency of the TBBU threshold falling with the ERL's top energy remains. The other important conjecture is that the TBBU threshold is proportional to the lowest electron beam energy passing through the ERL. Thus, the requirement of a high ERL beam current may contradict a natural desire to dump the beam on as low energy as possible.

\footnotetext{
${ }^{3}$ The geometric impedance, $R_{g}$, is a function of the cavity's geometry, and does not depend on the material (i.e., it does not depend on $Q$ ). However, often the rf literature contains a confusing form $R_{g}=R / Q$.
} 
It is noteworthy that, in modern TBBU theory, electron bunches are considered to be monoenergetic pointlike charges that act upon and react identically. Most of the TBBU codes (except that in [22]) assume this in simulating such instability.

The unfavorable scaling discussed above may have major implications on the cost of a high-energy ERL. Since an SRF linac is usually more expensive than magnetic elements, cost-effective solutions [22-25] lead to three- to six-pass ERLs. If the current in such ERL designs suffers from severe limitation, then the extent of their use and their energy reach will be restricted.

One way of resolving these confines lies in reducing the $Q$ of all dangerous HOMs by developing complex HOMdamping schemes, and in addition, circumscribing the number of cells per linac module so as to avoid trapping high- $Q$ HOMs [26-28]. These stringent requirements on HOM suppression frequently complicate the SRF linac designs, reduce the real-estate gradient, and make them more expensive.

The other way of increasing the threshold current is by lowering $\left\langle\left|T^{I J}\right|\right\rangle$. The latter is the topic of this paper, wherein I describe the workings of my proposed suppression mechanism.

\section{SUPPRESSION OF THE BEAM'S RESPONSE USING THE ERL LATTICE'S CHROMATICITY}

Since the bunch is an ensemble of particles, its transverse response to the kick will be the average if the responses of the individual particles are

$$
\left\langle x_{r}\right\rangle=\left\langle T_{12}\right\rangle \cdot x^{\prime}
$$

where $\langle a\rangle$ signifies the average value of a parameter, $a$. I suggest using the chromaticity of the returning loops and an energy spread in the beam (induced by an rf chirp, if needed) to reduce $\left\langle T_{12}\right\rangle \rightarrow 0$.

In contrast to storage rings wherein the beam circulates for trillions of turns, in an ERL the beam passes through the system only once on its way up in energy, and once on its way down. In storage rings, chromaticity must be compensated for assuring both the beam's stability and a reasonable lifetime; this is unnecessary in ERLs, and, as discussed in this paper, may even be counterproductive. Hence, I consider a linear ERL lattice without sextupole fields. $^{4}$

Let us consider the transverse motion of an ultrarelativistic particle with momentum $p=p_{o}(1+\delta) ;|\delta| \ll 1$ propagating through the linear transport system of the

\footnotetext{
${ }^{4}$ One might consider using nonlinear elements (sextupoles and octupoles) to introduce phase-advance dependence on betatron action, i.e., the square of the amplitude of betatron oscillations. While possibly this approach might be valid, such a method would require introducing a significant number of nonlinear field components on the scale of the transverse beam's size, and can negatively affect the beam's quality.
}

returning loop with a designed momentum, $p_{o}$, as is described by Hill's differential equation: ${ }^{5}$

$$
x^{\prime \prime}=\frac{d^{2} x}{d s^{2}}=-\frac{K_{1}(s)}{1+\delta} \cdot x \cong-(1-\delta) K_{1}(s) \cdot x,
$$

where $s$ is the longitudinal coordinate along the designed trajectory, and $K_{1}(s)$ is the rigidity of the transverse focusing for particles with the design energy [29]. The solution of this equation is well known in accelerator literature as the Courant-Snyder parametrization [30]:

$$
\begin{aligned}
x & =a \mathrm{w}(s) \cos (\psi(s)+\varphi) ; \quad \psi^{\prime}=1 / \mathrm{w}^{2} ; \\
x^{\prime} & =a \mathrm{w}^{\prime}(s) \cos (\psi(s)+\varphi)-\frac{a}{\mathrm{w}(s)} \sin (\psi(s)+\varphi),
\end{aligned}
$$

where $a$ and $\varphi$ are the constants of motion, and both the envelope function $\mathrm{w}(s, \delta)$ and $\psi(s, \delta)$ depend on the particle's momentum. ${ }^{6}$ Assuming the kick at $s=0$ as the initial condition, $x_{o}=0 ; x_{o}^{\prime}=x^{\prime}$, yields

$$
\begin{gathered}
\varphi=-\pi / 2 ; \quad a=\mathrm{w}_{\mathrm{i}} x^{\prime} ; \quad \mathrm{w}_{\mathrm{i}} \equiv \mathrm{w}(0) ; \\
x=x^{\prime} \cdot \mathrm{w}_{\mathrm{i}} \mathrm{w}(s) \sin \psi(s) .
\end{gathered}
$$

If all particles in the beam have the same energy, $E_{o}$, they will respond identically to the kick with $T_{12}(0 \mid \mathrm{s})=\mathrm{w}_{\mathrm{i}} \mathrm{w}(\mathrm{s}) \sin \psi(s){ }^{7}$ For a particle with a small energy deviation, $|\delta| \ll 1$, the envelope function and the phase advance can be extended as

$$
\begin{aligned}
& \mathrm{w}(s, \delta) \cong \mathrm{w}_{\mathrm{o}}(s)[1+\delta \cdot v(s)] ; \\
& \psi(s, \delta) \cong \psi_{\mathrm{o}}(s)+\delta \cdot \phi(s),
\end{aligned}
$$

where the subscript "o" indicates the values for particles with the designed energy. The straightforward perturbation theory (see Appendix A and C) yields the following expression:

$$
\begin{aligned}
& \phi(s)=-\frac{1}{2} \int_{0}^{s} K(z) \mathrm{w}_{0}^{2}(z)\left\{1+\cos 2\left[\psi_{o}(z)+\varphi_{o}\right]\right\} d z ; \\
& v(s)=-\frac{1}{2} \int_{0}^{s} K(z) \mathrm{w}_{0}^{2}(z) \cdot \sin 2\left[\psi_{o}(z)+\varphi_{o}\right] d z .
\end{aligned}
$$

One important (and well-known) feature of this solution is that the phase deviation (i.e., the chromaticity of oscillations) is defined by a monotonic function with average rate of $-\left\langle K(z) \mathrm{w}_{\mathrm{o}}^{2}(z)\right\rangle_{z} / 2$, while the envelope deviation is a fast oscillating function with double-betatron frequency.

\footnotetext{
${ }^{5}$ Throughout this paper I use the notations ${ }^{\prime} \equiv \frac{d}{d s} ; " \equiv \frac{d^{2}}{d s^{2}}$.

${ }^{6}$ I use the simple case of constant particle energy and momentum connected by a standard relativistic relation $E^{2}=p^{2} c^{2}+$ $m^{2} c^{4}$, see Appendix D and Refs. [29,31,32] for cases of arbitrary linear coupling and varying $e$-beam energy.

${ }^{7}$ The simple approach of choosing $\sin \psi(s)=0$ is possible only for a single cavity ERL. In practice, the length of the linacs, $L$, is large in any high-energy ERL linac $(L \sim 100 \mathrm{~m}$ for a $1 \mathrm{GeV}$ SRF linac), and the average value of $\left|T_{12}\right|$ is comparable to the linac's length, i.e., $\max \left(\left|T_{12}\right|\right)_{\text {linac }} \sim L \sim 1 / N$.
} 
Hence, in a large accelerator system, chromaticity grows steadily, and can reach significant values:

$$
C(s)=\frac{\phi(s)}{2 \pi} \gg 1,
$$

and, even for a modest deviation in relative energy, phase variation can be large, while the relative variation in the envelope function remains small:

$$
\delta \cdot v(s) \ll 1 .
$$

The latter assures the absence of emittance growth in chromatic transport line for a matched beam. It is noteworthy that chromatic transport would cause emittance growth for mismatched injected beam with that growth being proportional to the mismatch (details are given in Appendix C).

Let us consider a bunch with Gaussian energy distribution with a relative rms energy spread $\sigma_{\delta}:{ }^{8}$

$$
f(\delta)=\frac{1}{\sqrt{2 \pi} \sigma_{\delta}} \exp \left(-\frac{\delta^{2}}{2 \sigma_{\delta}^{2}}\right) .
$$

We then calculate the value of the electron bunchs' displacement, i.e., $\langle x\rangle$ :

$$
\langle x(s)\rangle=x^{\prime} \cdot \mathrm{w}_{\mathrm{i} 0} \int_{-\infty}^{\infty} \mathrm{w}(s, \delta) \sin \psi(s, \delta) f(\delta) d \delta .
$$

Substituting (10), the integral (15) is evaluated readily:

$$
\begin{aligned}
\left\langle T_{12}\right\rangle= & \frac{\langle x(s)\rangle}{x^{\prime}} \\
= & \exp \left(-\frac{\left(\phi \sigma_{\delta}\right)^{2}}{2}\right) \cdot \mathrm{w}_{\mathrm{io}} \mathrm{w}_{\mathrm{o}}(s)\left[\sin \psi_{\mathrm{o}}(s)\right. \\
& \left.+v(s) \phi(s) \sigma_{\delta}^{2} \cos \psi_{\mathrm{o}}(s)\right],
\end{aligned}
$$

with the suppression factor exponentially dependent on the product of the chromaticity and the energy spread:

$$
\left\langle T_{12}\right\rangle \propto \exp \left(-\frac{\left(\phi \sigma_{\delta}\right)^{2}}{2}\right) \cdot T_{12}(\max ) .
$$

Figures 3 and 4, below, demonstrate in its simplest form the effect of chromaticity on the response of the electron beam to a transverse-momentum kick. Section IV gives more detailed examples.

Assuming a strong focusing lattice for return loops comprised of many periodic cells, similar to that designed for the eRHIC electron-hadron collider [24], the loops' chromaticity can be $C(s) \sim-300$ and $\phi(s) \sim 2 \times 10^{3}$. Then, for a Gaussian beam with an rms energy spread of

\footnotetext{
${ }^{8} \mathrm{We}$ can produce such a beam in an ERL injector from a cold beam generated from photoinjectors. For example, by creating an electron bunch at the photocathode with a Gaussian longitudinal profile, and then accelerating it off-crest will assure the desirable distribution. Section III details this method.
}

(a)

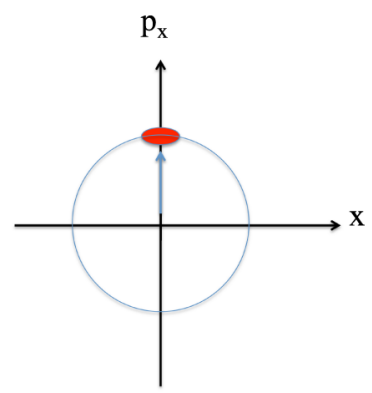

(b)

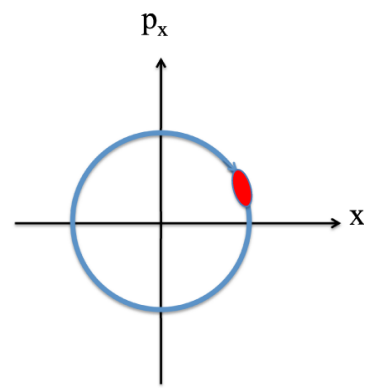

FIG. 3. In an arc with zero chromaticity, a transversemomentum kick received from the linac (a), will translate (after rotation in the phase space) into a displacement of the entire bunch at the exit of the transport channel (arc) (b).

$0.2 \%$, the response $\left\langle T_{12}\right\rangle$ will be suppressed 3000 -fold, and, according to formula (2), the threshold for TBBU instability will increase by about this value, or will be limited by another type of instability.

While chromaticity exponentially suppresses the dipole moment excited in the beam by a kick, it does not cause the beam's emittance to grow; details are given in Appendix C.

Naturally, the level of suppression of the beam's response (transfer function, $\left\langle T_{12}\right\rangle$ ) depends on the energydistribution function; Table I gives a few examples.

Smooth bell-shaped energy-distribution functions (Gaussian, Lorentzian, $\kappa-2$ ) assure the strong suppression of $\left\langle T_{12}\right\rangle$ whose value falls fast with the increase of $|X| \equiv 2 \pi|C| \sigma_{\delta}$; sharp-edged distribution functions (such as rectangular or triangular ones) cause the oscillating behavior of $\left\langle T_{12}\right\rangle$ which declines more slowly with the growth of $|X|$. In all cases, a highly chromatic lattice resulting in $|X| \gg 1$ effectively suppresses the beam's response to transverse kicks. (a)

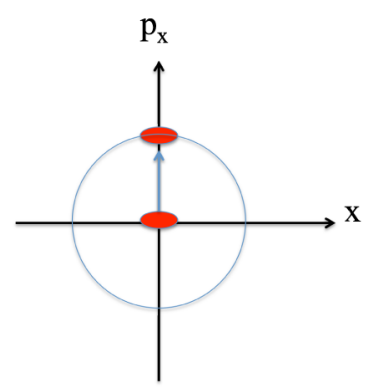

(b)

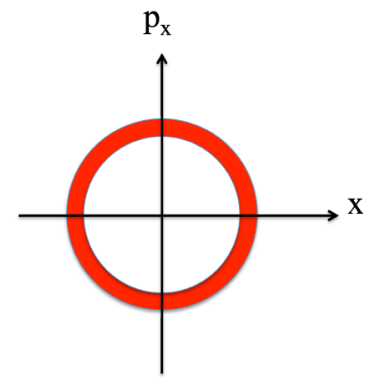

FIG. 4. In an arc with large product of the chromaticity and energy spread, a transverse-momentum kick received from linac (a) will not displace the bunch overall because the dependence of the phase-space rotation angle on the particles' energy spreads their displacement evenly around the circle (b). 
TABLE I. Suppression of the beam's response on a transverse kick by the chromaticity, $C$. In this table $X=\phi \sigma_{\delta} ; Y=v \sigma_{\delta}$.

\begin{tabular}{llr}
\hline \hline$f(\delta)$ & Suppression factor $\left\langle T_{12}\right\rangle / \mathrm{w}_{\mathrm{io}} \mathrm{w}_{\mathrm{o}}$ & Type \\
\hline$\frac{1}{\sqrt{2 \pi} \sigma_{\delta}} \exp \left(-\frac{\delta^{2}}{2 \sigma_{\delta}^{2}}\right)$ & $e^{-X^{2} / 2} \cdot(\sin \psi+X \cdot Y \cos \psi)$ & Gaussian \\
$\frac{1}{\pi \sigma_{\delta}}\left(1+\frac{\delta^{2}}{\sigma_{\delta}^{2}}\right)^{-1}$ & $e^{-|X|} \cdot(\sin \psi+Y \cdot \operatorname{sign}(X) \cos \psi)$ & Lorentzian \\
$\frac{2}{\pi \sigma_{\delta}}\left(1+\frac{\delta^{2}}{\sigma_{\delta}^{2}}\right)^{-2}$ & $e^{-|X|} \cdot((1+|X|) \sin \psi+X \cdot Y \cos \psi)$ & $\kappa-2$ \\
$\frac{1}{2 \sigma_{\delta}}\left(\begin{array}{c}\theta\left(\delta-\sigma_{\delta}\right)- \\
\theta\left(\delta+\sigma_{\delta}\right)\end{array}\right)$ & $\frac{\sin X}{X} \sin \psi+Y \frac{\sin X-X \cos X}{X^{2}} \cos \psi$ & Rectangular \\
$\frac{\left|1-\delta / \sigma_{\delta}\right|}{\sigma_{\delta}},|\delta| \leq \sigma_{\delta}$ & $2\left(\frac{\cos X-1}{X^{2}} \sin \psi+Y \cdot \frac{2(\cos X-1)+X \sin X}{X^{3}} \cos \psi\right)$ & Triangular \\
\hline \hline
\end{tabular}

\section{PRACTICAL ISSUES OF DEPHASING}

In many cases, there is insufficient chromaticity in an arc and energy spread in the electron beam to suppress the beam's response. For example, most presently designed ERLs for future light sources and their prototypes [33-36] have a relatively weak focusing lattice and, therefore, low chromaticity. Furthermore, there is a tendency to use sextupoles in compensating for natural chromaticity. Hence, in several cases the naturally occurring energy spread is insufficient to measurably suppress the beam's response. In addition, for most ERLs it is beneficial to keep the energy spread of the beam as low as possible. ${ }^{9}$

Hence, it would be natural to create such a response suppressor artificially using two rf cavities: one to create an energy spread at the entrance of the arc and the other to remove it at its end (Fig. 5). Since most ERLs use isochronous arcs (i.e., arcs with $T_{56}=0$ ), neither the energy spread nor the beam's bunch length are affected by such a system. The only effect will be to introduce additional energy spread in the arc, thereby suppressing the beam's response.

There are many advantages to such a system. One is that electron beams generated from photoinjectors frequently have a Gaussian longitudinal distribution, and a linear chirp in the rf cavity will introduce a Gaussian energy spread in the beam. This means that, for $2 \pi C \sigma_{\delta} \gg 1$, the beam's response would be completely (exponentially) suppressed.

This scheme affords us a practical way to suppress TBBU instability in almost all ERLs. Using an energy chirp in the part of the arcs where energy spread is allowed suppresses the beam's response, while removing of this

\footnotetext{
${ }^{9}$ For the eRHIC's ERL with 6 passes [8], the energy of the electrons will affect its spin precession, and preserving the polarization of the electron beam would require alternating the sign of the chirp on consecutive passes. Since one arc in eRHIC is twice the length of the other, and therefore, has a chromaticity twice as large, using a chirp of the same amplitude would keep most of the "memory loss" experienced in the previous section. In the $\mathrm{LHeC}$ case, the vertical spin direction will be retained in the arcs. Accordingly, this scheme can be used without alternating the chirp's sign.
}

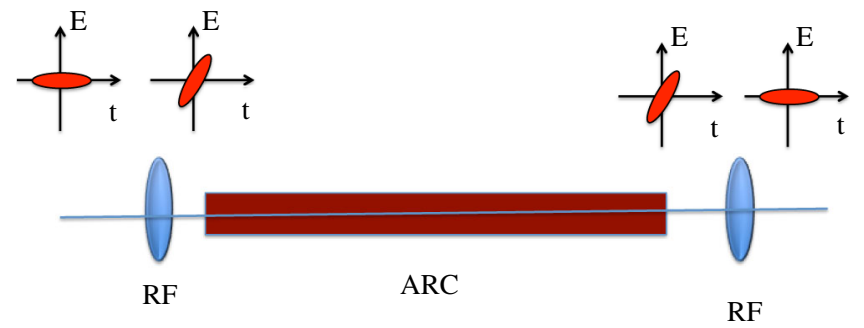

FIG. 5. A sketch of a dedicated TBBU suppressor: One rf cavity gives an energy chirp to the electron beam at the entrance to the arc, and the other cavity removes the chirp.

energy chirp returns the energy spread to its natural value before using the beam (for example, in an undulator or in a collision). In such a scheme, the local energy spread in the bunch remains low.

\section{EXAMPLES}

Let us consider two specific examples of multipass ERLs proposed for the electron-hadron colliders, eRHIC and $\mathrm{LHeC}$. Two recent papers describe their low-emittance achromatic arc lattices $[37,38]$.

eRHIC's $30 \mathrm{GeV}$ ERL, shown in Fig. 6, comprises a $0.6 \mathrm{GeV}$ injector and two $2.45 \mathrm{GeV}$ superconducting rf linacs. All eRHIC linacs work in the energy recovery mode; the injector is a single-path ERL, while the main linacs are part of a 6-pass ERL. The electron beam from a $10 \mathrm{MeV}$ preinjector is accelerated to $0.6 \mathrm{GeV}$ in the injector ERL and then accelerated further to $30 \mathrm{GeV}$, passing six times through the main SRF linacs. After collision in one of eRHIC's detectors, the electron beam is decelerated to $10 \mathrm{MeV}$ and then is dumped. The path length of the $30 \mathrm{GeV}$ beam line provides for 180-degree phase shift, and the used electrons decelerate at the same linacs in the reverse sequence. After reaching $0.6 \mathrm{GeV}$, they are routed to the injection ERL to recover their energy.

eRHIC's low-emittance ERL lattice has natural chromaticity of $C_{x_{s}}=-28.571 ; C_{y_{s}}=-20.242$ per sextant. While the mergers and combiners, as well as the straight 


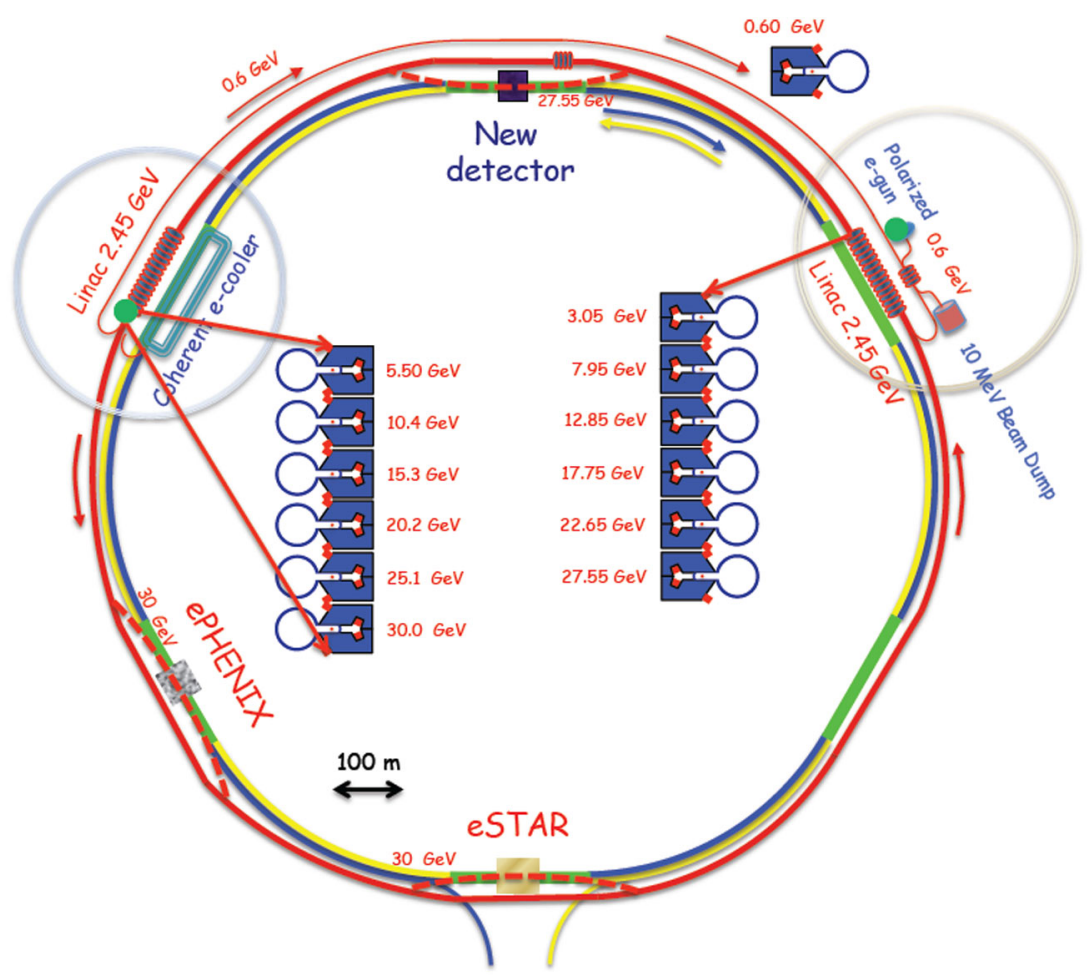

FIG. 6. A sketch of the eRHIC with $30 \mathrm{GeV}$ ERL. More details about the eRHIC's design are given in [24,37].

sections, add to natural chromaticity, their contribution is relatively small, i.e., just few units. Accordingly, the total $360^{\circ}$ turn chromaticities can be estimated as $C_{x_{t}} \cong$ $-175 ; \quad C_{y_{t}} \cong-125$, and $\phi_{x_{t}} \approx 1100 ; \quad \phi_{y_{t}} \approx 800 .^{10}$ Furthermore, total accumulated chromaticity for six passes up in energy and five passes down is $C_{x_{\mathrm{ERL}}} \sim-2000$; $C_{y_{\mathrm{ERL}}} \sim-1400$ and $\phi_{x_{\mathrm{ERL}}} \approx 1.25 \times 10^{4} ; \quad \phi_{y_{\mathrm{ERL}}} \approx$ $8.5 \times 10^{3}$.

Figure 7 shows BNL's version of $60-\mathrm{GeV}$ ERL for $\mathrm{LHeC}$ electron-hadron collider. It comprises a single-pass $0.3 \mathrm{GeV}$ energy recovery injector, and two main $9.95 \mathrm{GeV}$ superconducting rf linacs. Electrons pass three times through the main linacs on their way up to $60 \mathrm{GeV}$, and, after colliding with LHC protons, three times on the way down in energy.

The low-emittance ERL lattice for this ERL has natural chromaticity of $C_{x_{s}}=-254.29 ; C_{y_{s}}=-227.37$ per $180^{\circ}$ arc. Similarly to eRHIC's ERL lattice, the mergers, combiners, and straight sections add just a few units to natural chromaticity. The chromaticities of the $360^{\circ}$ turn are $C_{x_{t}} \cong-510 ; \quad C_{y_{t}} \cong-460$, and $\varphi_{x_{t}} \approx 3.2 \times 10^{3}$; $\phi_{y_{t}} \approx 2.9 \times 10^{3}$. Similarly, the total accumulated chromaticity on three passes up and two passes down is

\footnotetext{
${ }^{10}$ The chromaticity is properly defined for a beam with constant energy, $E$, with $\delta=\delta E / E$ being constant. In linacs where the energy changes, and $\delta E$ is no longer a constant, traditional chromaticity should be redefined.
}

$C_{x_{\mathrm{ERL}}} \sim-2500 ; C_{y_{\mathrm{ERL}}} \sim-2300$ and $\phi_{x_{\mathrm{ERL}}} \approx 1.5 \times 10^{4}$; $\phi_{y_{\mathrm{ERL}}} \approx 1.4 \times 10^{4}$.

In contrast to the cumulative beam breakup, the TBBU ERL instability relies on the feedback provided by electron bunches returning to the same rf cavity. In other words, such feedback occurs only after $e$-beam goes through a complete 360-degree turn. Hence, one-turn chromaticity plays a dominant role in suppressing a single-turn TBBU.

To roughly estimate TBBU suppression in the above ERLs, I consider an electron beam with an induced

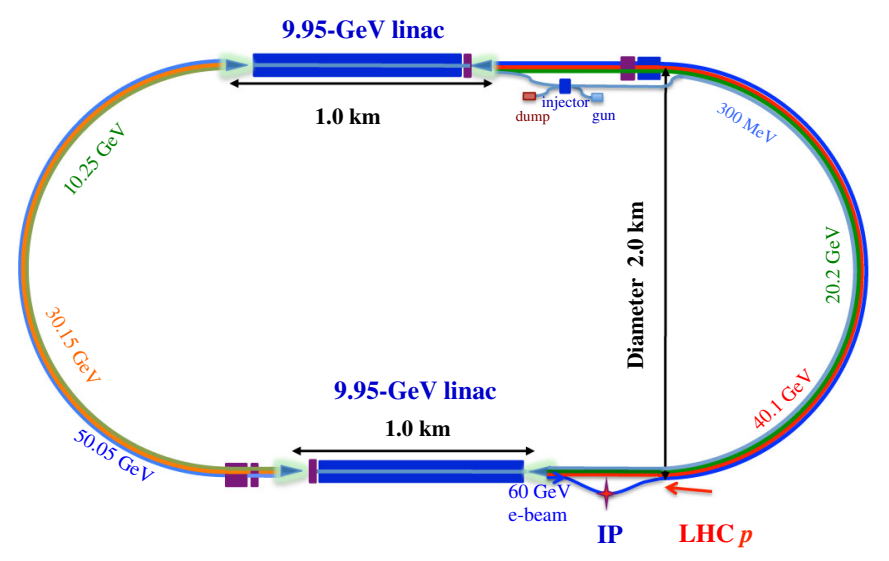

FIG. 7. BNL's design of $60 \mathrm{GeV}$ ERL for the $\mathrm{LHeC}$ linac-ring collider [47]. 
TABLE II. Propagation of electron bunch in the eRHIC ERL with $\sigma_{E}=0.025 \mathrm{GeV}{ }^{\mathrm{a}}$

\begin{tabular}{|c|c|c|c|c|}
\hline Element & Energy in, GeV & Energy out, GeV & $\Delta \psi_{x} @ \sigma_{E}$ & $\Delta \psi_{y} @ \sigma_{E}$ \\
\hline Linac 1 & 0.6 & 3.05 & -0.104247483 & -0.104247483 \\
\hline Arc 1 & 3.05 & 3.05 & -1.471449897 & -1.042493746 \\
\hline Linac 2 & 3.05 & 5.5 & -0.037802111 & -0.037802111 \\
\hline Arc 2 & 5.5 & 5.5 & -0.815985852 & -0.578110168 \\
\hline Linac 1 & 5.5 & 7.95 & -0.023621176 & -0.023621176 \\
\hline Arc 3 & 7.95 & 7.95 & -0.564518514 & -0.399950431 \\
\hline Linac 2 & 7.95 & 10.4 & -0.017223229 & -0.017223229 \\
\hline Arc 4 & 10.4 & 10.4 & -0.431530979 & -0.305731339 \\
\hline Linac 1 & 10.4 & 12.85 & -0.013562576 & -0.013562576 \\
\hline Arc 5 & 12.85 & 12.85 & -0.349254645 & -0.24744015 \\
\hline Linac 2 & 12.85 & 15.3 & -0.011188495 & -0.011188495 \\
\hline Arc 6 & 15.3 & 15.3 & -0.293328247 & -0.207817381 \\
\hline Linac 1 & 15.3 & 17.75 & -0.009523045 & -0.009523045 \\
\hline Arc 7 & 17.75 & 17.75 & -0.252840686 & -0.179132728 \\
\hline Linac 2 & 17.75 & 20.2 & -0.008289771 & -0.008289771 \\
\hline Arc 8 & 20.2 & 20.2 & -0.222174366 & -0.157406234 \\
\hline Linac 1 & 20.2 & 22.65 & -0.00733961 & -0.00733961 \\
\hline Arc 9 & 22.65 & 22.65 & -0.19814226 & -0.140379953 \\
\hline Linac 2 & 22.65 & 25.1 & -0.006585034 & -0.006585034 \\
\hline Arc 10 & 25.1 & 25.1 & -0.178801681 & -0.126677527 \\
\hline Linac 1 & 25.1 & 27.55 & -0.00597125 & -0.00597125 \\
\hline Arc 11 & 27.55 & 27.55 & -0.162900987 & -0.115412193 \\
\hline Linac 2 & 27.55 & 30 & -0.005462194 & -0.005462194 \\
\hline Arc 12 & 27.55 & 30 & -0.005462194 & -0.005462194 \\
\hline Linac 1 & 30 & 30 & -0.146264073 & -0.102653531 \\
\hline
\end{tabular}

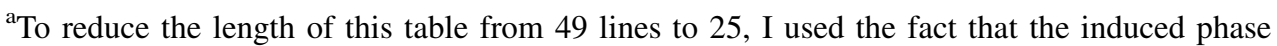
spread is identical in each arc in linac on the way up and on the way down in energy. Except for the $30 \mathrm{GeV}$ arc (and injection/ejection lines), the bunch passes each other element of the ERL twice. I note that arcs with odd number comprise two sextants, while four sextants comprise arcs with even numbers.

Gaussian energy spread of $\sigma_{\delta}=5 \times 10^{-3} \cdot{ }^{11}$ For eRHIC's ERL, the vertical response of the bunch (i.e., a one-turn response $\left\langle T_{12}(s|s+C\rangle)\right.$ will be suppressed by a factor of $2.9 \times 10^{3}$, while the horizontal suppression factor will be an astronomic factor of $3.7 \times 10^{6}$. Since the chromaticity per turn is higher in the case of $\mathrm{LHeC}$, the suppression will be astronomical factor of $\sim 10^{45}$, i.e., other higher order effects and the imperfections of the Gaussian profile should be considered. It also means that for the LHeC, $\sigma_{\delta} \cong$ $1.3 \times 10^{-3}$ will suffice for suppressing a single-turn response by a factor of 1000 .

It is noteworthy that some ERLs proposed for future light sources have relatively large chromaticity. For example, the 7-GeV arc of the proposed ERL at Argonne National Laboratory [36] would have a chromaticity $\sim \phi_{\text {arc }} \approx 380$; introducing energy chirps with a relative

\footnotetext{
${ }^{11}$ As discussed above, such energy spread can be correlated and introduced intentionally by off-crest acceleration of the electron bunch with a Gaussian longitudinal profile. Furthermore, if needed, the sign of the energy chirp can be alternated from pass to pass. The latter action does not affect the conclusions.
}

rms energy spread of $0.5 \%$ would suppress the beam's response sixfold. Even though this level is less impressive than that for the eRHIC and LHeC ERLs, it would support the usage of a multiturn ERL for such a light source, and, potentially, significantly lower its cost. In addition, it may be economically viable to increase the number of arc cells and corresponding chromaticities in such ERL to allow a multipass scheme.

Very large values of accumulated chromaticity $\phi \propto$ $-10^{4}$ for a beam passing through the entire eRHIC or LHeC ERL means that an average $\sigma_{\delta} \sim 5 \times 10^{-4}$ will erase the memory of a low beam returning to the ERL injector, i.e., to suppress $\left\langle T_{12}\right.$ (in $|$ out $\left.)\right\rangle$ of the main ERL by three to four orders of magnitude.

To improve the accuracy of this statement, I consider here an electron bunch propagating through the entire eRHIC ERL [24,37], starting at an injection energy of $0.6 \mathrm{GeV}$, accelerating to $30 \mathrm{GeV}$, and then decelerated back to $0.6 \mathrm{GeV}$, traversing eleven times around the RHIC tunnel. I consider inducing a linear (correlated) energy chirp on a Gaussian electron bunch with an rms value of $0.025 \mathrm{GeV}$ at the exit of the injection channel 
(a)

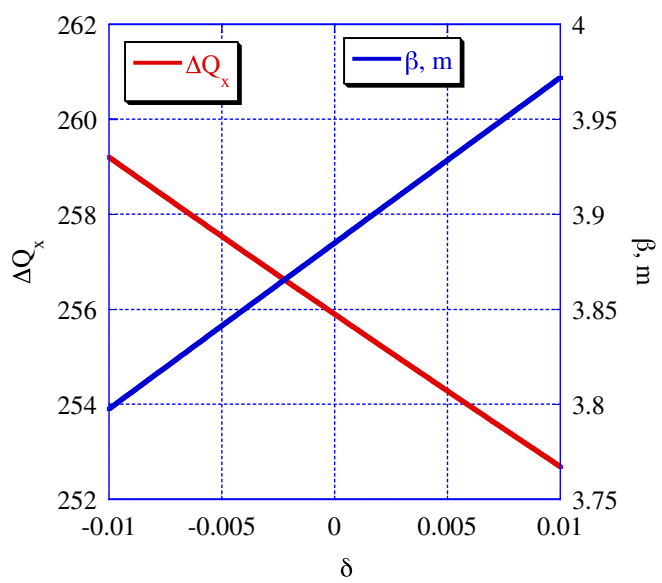

(b)

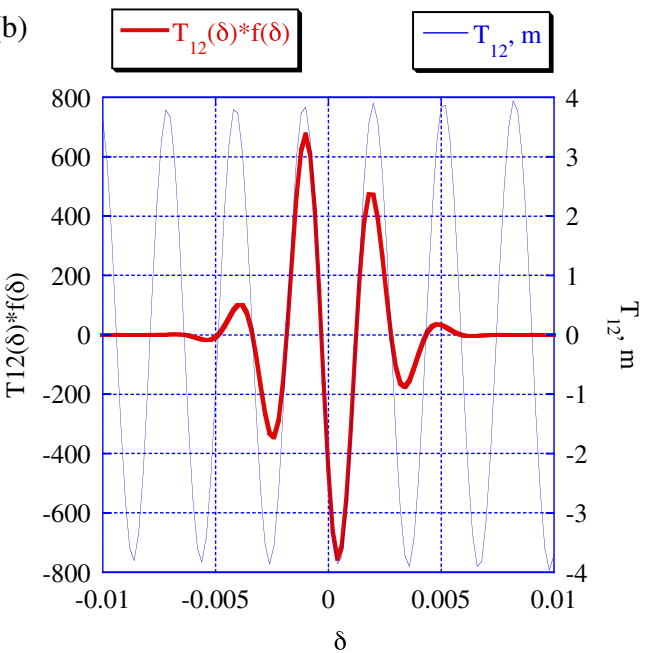

FIG. 8. The FODO beam line optics parameters as a function of the particle momentum for a nominal tune advance per cell $\Delta Q_{x}(\delta=0)=0.2499$ : (a) dependence of the total horizontal tune advance (red line), and beta function (blue line) on the relative momentum deviation $\delta$; (b) dependence of the beam line's $T_{12}$ element (blue line) and its convolution with the momentum distribution function (red line) on the relative momentum deviation $\delta$.

(and taking this chirp out from the ejecting beam before it goes through ejection beam line). For simplicity, I neglect the natural energy spread from the gun, along with that accumulated from the quantum fluctuation of synchrotron radiation in eleven passes, since these effects would only increase suppression.

Since the eRHIC arcs have an identical lattice in all arcs, the arcs have zero values of $T_{56}$ [37], and since electrons are accelerated on-crest, their longitudinal motion is frozen and energy deviation at every pass is determined. Thus, we can calculate exactly the accumulated spread of the betatron-oscillation phases caused by the induced energy spread. Table II gives details of the $e$-beam propagating through eRHIC, and the accumulated betatron-phase spreads. Intuitively it is understandable that the betatron phase is additive, i.e., it accumulates at every element of the accelerator's transport channel. Appendix D gives the theoretical foundation of this approach.

Simple arithmetic reveals in this case that the induced phase spreads will be 10.53 and 7.61 in the horizontal and vertical planes, correspondingly. The exponential term (17) with $X=7$ or $X=10$ would represent astronomic suppression (i.e., 12 to 24 orders of magnitude!) in the beam's response at the exit of the eRHIC ERL on the kick at its entrance. The exact value of $Y$ (which is in the order of unity-see example in Fig. 8) would not change this conclusion, and, in practice, the deviation of the beam from the Gaussian form, or higher order effects will determine the beam's residual response.

It also means that a chirp with rms of $0.0125 \mathrm{GeV}$ would be sufficient suppress the round-trip beam response by 3 orders of magnitude.

Thus, the standard conjecture from modern TBBU theory that in an $N$-pass ERL the lowest value of the beam energy will determine the threshold as well as $N^{-2}$ scaling of the TBBU threshold current would not apply for the case discussed herein. Hence, a multipass ERL approach for light sources, similar to that proposed by Budker Institute of Nuclear Physics [39], can take advantage of this suppression mechanism.

\section{NUMERIC EXAMPLE}

As discussed in Appendix C, some higher order effects can, in principle, reduce or at least significantly modify the prediction of Eq. (17). To address this concern, I tested the concept by directly calculating the response of the beam with a Gaussian energy distribution propagating through a beam line encompassing 1024 FODO cells. Each FODO cell comprises one focusing (F) and one defocusing (D) quadrupole separated by a drift spaces $(\mathrm{O})$.

Hence, I use a paraxial approximation for the particle's trajectory, so that it is easy to write the exact analytical expression for the transport matrix of the FODO cell for an ultrarelativistic particle with arbitrary momentum $p=$ $p_{o}(1+\delta): T_{\mathrm{FODO}}(\delta)$. The matrix is multiplied by the necessary number of times ${ }^{12}$

$$
T_{\text {total }}(\delta)=\left[T_{\mathrm{FODO}}(\delta)\right]^{1024}
$$

to form the exact matrix of the beam line. The beam's displacement at the end of the beam line to an angular kick, $\theta$, at its entrance entails a simple convolution:

\footnotetext{
${ }^{12}$ The number of cells, $N=1024=2^{10}$ was chosen to shorten the simulation time by reducing $N$ matrix multiplications to $\log _{2}(N)=10$ via $T^{2^{n+1}}=\left[T^{2^{n}}\right]^{2}$.
} 


$$
\begin{aligned}
\langle x\rangle & =\left\langle T_{12 \text { total }}\right\rangle \cdot \theta ; \\
\left\langle T_{12 \text { total }}\right\rangle & =\int_{-\infty}^{\infty} T_{12 \text { total }}(\delta) f(\delta) d \delta,
\end{aligned}
$$

where $T_{12 \text { total }}(\delta)$ is an exact, analytical, nonlinear function of $\delta$. For comparison, I define the suppression factor of the transverse response as

$$
S=\frac{\max \left(\left|T_{12 \text { total }}(\delta)\right|\right)}{\left|\int_{-\infty}^{\infty} T_{12 \text { total }}(\delta) f(\delta) d \delta\right|}
$$

Here, I present results for a FODO lattice with equal focusing in both directions. ${ }^{13}$ The exact transverse matrix of such a FODO cell, comprised of two quadrupoles with length $l$, and the nominal strength $K_{1}$ and separated by two drift spaces with length $L$ is the following:

$$
\begin{gathered}
T_{\mathrm{FODO}}(\delta)=\left[\begin{array}{cc}
1 & L \\
0 & 1
\end{array}\right] \cdot\left[\begin{array}{cc}
\cos \phi & \kappa^{-1} \sin \phi \\
-\kappa \sin \phi & \cos \phi
\end{array}\right] \cdot\left[\begin{array}{cc}
1 & L \\
0 & 1
\end{array}\right] \cdot\left[\begin{array}{cc}
\cosh \phi & \kappa^{-1} \sinh \phi \\
\kappa \sinh \phi & \cosh \phi
\end{array}\right] ; \quad \kappa(\delta)=\sqrt{\frac{K_{1}}{1+\delta}} \\
\phi(\delta)=\kappa(\delta) l .
\end{gathered}
$$

This exact matrix function was used for direct numerical calculations of $T_{12 \text { total }}(\delta)$ of the beam line, and convolving it with Gaussian energy distribution (14) with relative rms energy spread of $\sigma_{\delta}=2 \times 10^{-3}$. I did not observe any significant deviations from the expected theoretical behavior predicted by Eq. (17) either at a small values (as expected) or at large values of the of total beam line's chromaticity.

For example, the beam line with tune advance of 0.22285 per cell has chromaticity of $C=-276.68$, and the calculated suppression factor of 428 -fold that compares favorably with the theoretical estimation of 422-fold given by Eq. (17).

Increasing the chromaticity further to $C=-328.51$ by increasing the tune advance per cell to 0.2499 increased the calculated suppression factor to $5.93 \times 10^{3}$. This also is reasonably close to the theoretical estimation of $5.02 \times 10^{3}$ given by Eq. (17).

It is remarkable that, at large values of the suppression, the exact value of the suppression of the beam's response exceeds the theoretical predictions. A possible explanation of this phenomena is that high-order effects increase the particle's decoherence compared with firstorder chromatic effects. As evident from Fig. 8, the behavior of the chromatic suppression does not deviate strongly from first-order chromatic behavior represented by Eqs. (10) and (11).

The tune advance demonstrates mostly linear dependence on $\delta$ with the quadratic term contributing $\Delta Q_{x}^{\text {snd }} \cong 0.049$ at $\delta= \pm 0.01$. As expected, neither the $\beta$ function nor the envelope of $T_{12}$ significantly change within the $\delta= \pm 0.01$ range. The $\beta$ function changes only for less than $1 \%$ at $\delta=\sigma_{\delta}$, and a smooth envelope of oscillating $T_{12}$ provides for a strong suppression of the overall response when convoluted with smooth energy distributions.

\section{DISCUSSION AND CONCLUSIONS}

I have shown in this paper that using the natural chromaticity of the arcs in ERLs affords an opportunity of suppressing the beam's transfer function (its response to transverse kicks), and either suppressing TBBU instability or significantly increasing allowable operating currents. It is important here to note that using chromatic arcs requires matching the injected beam into the ERL lattice to preserve the beam's emittance since that of an mismatched beam can increase.

In an ERL comprising a sequence of linacs and arcs, with the product of the chromaticity and the energy spread in the $\operatorname{arcs}\left|\phi \sigma_{\delta}\right| \gg 1$, the electron beam forgets the kick it received in the linacs while traveling through an arc. Then, the traditional ERL TBBU excitation scheme falls apart, and each linac effectively sees fresh electron beams. Accordingly, the problem of the beam's stability could be reduced to the traditional cumulative transverse instability in long linacs. The nature of this instability [40] that differs from that of the ERL TBBU is outside the scope of this paper. It is well studied [41-43] and known to have higher instability threshold compared with that of the TBBU.

In the TBBU theory [14-19], the low-energy passes through the linac are considered to be the ones most vulnerable to TBBU instability. Specifically, the conclusions are that the TBBU threshold is proportional to the lowest energy in the recirculating path, and also inversely proportional to the number of the recirculating passes. The examples described in this paper show that this rule is not applicable to an ERL wherein the accumulated chromatic phase spread is significantly larger than unity. Hence, the proposed method may pave the way for multipass ERLs becoming an economic choice for high-energy electron accelerators. Thus, using highly chromatic lattices in ERLs may support cost-effective multipass ERLs, as well as very effective energy recovery attained by dumping the used electron beams at very low energies.

Using the natural chromaticity of the ERL lattice may offer a winning case: Thus, sextupole magnets are not required to compensate for chromaticity (i.e., reducing

\footnotetext{
${ }^{13}$ Tests with nonequal strength of the quadrupole did not reveal any difference in behavior from this example.
} 
the cost of the accelerator) while the threshold for TBBU instability goes up. Still, in special cases of compact machines wherein natural chromaticity is small, or when using a lattice with large natural chromaticity is impractical, installing sextupoles and intentionally increasing chromaticity to the desirable level might be considered. Compared with storage rings, the fact that in ERLs electrons are used only once provides much-relaxed requirements on the linearity of the lattice, and using very strong sextupoles could be possible.

In contrast to other mitigation methods, such as HOM damping, this method is straightforward and modern accelerator codes easily can simulate lattice effect on the electron beam's emittance. It also may allow us to use longer SRF linac strings, or an increase in the realestate gradients in ERLs, and lower injection energy. Overall, this method offers an additional tool in advancing ERL technology towards high-energy, high-current operations.

Standard TBBU simulation codes presently cannot encompass chromatic effects in the simulation, with one possible exception [22]. While verifying the proposed suppression mechanism by direct TBBU simulations would be beneficial, such tests are beyond the scope of this paper.

There was an experimental observation of about $60 \%$ TBBU threshold increase in JLab's ERL when FEL was lasing [44]. One plausible explanation of the increase is that the transverse beam response was chromatically suppressed by a very significant energy spread induced by the FEL lasing. There could be other plausible explanations of this phenomena and a detailed analysis is needed to identify the reason.

\section{ACKNOWLEDGMENTS}

The author would like to thank Ilan Ben-Zvi, Yue Hao, Dmitry Kayran (BNL), and Eduard Pozdeyev (FRIB) for fruitful, in-depth discussions of the methods, and Georg Hoffstaetter (Cornel University) and Frank Zimmermann (CERN) for their interest in the prospects of suppressing TBBU in high-energy ERLs. I am extremely grateful to Avril Woodhead (BNL) for editing the text of this paper and for providing excellent suggestions on the layout. The work was supported by Brookhaven Science Associates under Contract No. DE-AC02-98CH10886 with the U.S. DOE.

\section{APPENDIX A: ENVELOPE AND PHASE-ADVANCE DEPENDENCE ON THE PARTICLE ENERGY}

Here, I give a very short $1 \mathrm{D}$ derivation of the envelope and phase variation for an off-momentum particle. More details appear in $[31,32]$.

Let us consider the Hamiltonian of a transverse 1D linear motion of a particle with constant longitudinal momentum $p=p_{o}(1+\delta)$ in a beam line with a focusing function $K(s):^{14}$

$$
\mathbf{H}=\frac{p_{x}^{2}}{2}+\frac{K(s)}{1+\delta} \frac{x^{2}}{2}=\mathbf{H}_{o}-\nu K(s) \frac{x^{2}}{2},
$$

where $\nu=\delta /(1+\delta)$, which I assume to be infinitesimally small $|\nu| \cong|\delta| \ll 1$, and $p_{o}$ is the momentum of the reference particle. I use a known Courant-Snyder parametrization for the reference particle [30],

$$
\begin{aligned}
x & =a \cdot \mathbf{w}_{o}(s) \cdot \cos \left[\psi_{o}(s)+\varphi\right] ; \\
\beta_{o} & \equiv \mathbf{w}_{o}^{2} ; \quad \psi_{o}^{\prime}=1 / \beta_{o},
\end{aligned}
$$

where $a=\sqrt{2 I}, \varphi$ are determined by initial conditions. It is well known [45] that parametrization (A2) is a canonical transformation from $\left\{x, x^{\prime}\right\}$ to the action-angle variables $\{\varphi, I\}$ with reduced Hamiltonian:

$h=\mathbf{H}-\mathbf{H}_{o}=-\nu I \cdot K(s) \mathbf{w}_{o}^{2}(s) \cdot \cos ^{2}\left[\psi_{o}(s)+\varphi\right]$,

and reduced equations of motion:

$$
\begin{aligned}
\varphi^{\prime} & =\frac{\partial h}{\partial I}=-\nu \cdot K(s) \mathbf{w}_{o}^{2}(s) \cdot \cos ^{2}\left[\psi_{o}(s)+\varphi\right] \\
I^{\prime} & =-\frac{\partial h}{\partial \varphi}=-\nu \cdot I \cdot K(s) \mathbf{w}_{o}^{2}(s) \cdot \sin \left\{2\left[\psi_{o}(s)+\varphi\right]\right\} .
\end{aligned}
$$

Using the standard perturbation method [46] these equations are easily integrated:

$$
\begin{aligned}
\varphi(s)= & \varphi_{o}-\delta \cdot \int_{o}^{s} K(z) \mathbf{w}_{o}^{2}(z) \cdot \frac{1+\cos \left\{2\left[\psi_{o}(z)+\varphi_{o}\right]\right\}}{2} d z \\
& +O\left(\delta^{2}\right) \\
I= & I_{o}\left\{1-\delta \cdot \int_{o}^{s} K(z) \mathbf{w}_{o}^{2}(z) \cdot \sin \left\{2\left[\psi_{o}(z)+\varphi_{o}\right]\right\} d z\right\} \\
& +O\left(\delta^{2}\right),
\end{aligned}
$$

This result demonstrates that in a periodic lattice [i.e. $\left.K(s)=K(s+C) ; \mathrm{w}_{o}(s+C)=\mathrm{w}_{o}(s)\right]$ the envelope (amplitude) variation has only an oscillating term (traditionally called a beta beating), while the phase has both monotonically growing and oscillating terms. Substituting (A5) into (A2) and keeping only first-order terms of $\nu$ gives

$$
\begin{aligned}
x \cong & a_{0} \cdot \mathbf{w}_{\varepsilon}(s) \cdot \cos \left[\psi_{o}(s)+\varphi(s)\right] ; \\
\mathbf{w}_{\delta}(s)= & \mathbf{w}_{o}(s)\left\{1-\frac{\delta}{2} \cdot \int_{o}^{s} K(z) \mathbf{w}_{o}^{2}(z) \cdot \sin \left\{2\left[\psi_{o}(z)+\varphi_{o}\right]\right\} d z\right\} \\
& +O\left(\delta^{2}\right) .
\end{aligned}
$$

The choice of the initial amplitude and phase gives the required expressions in Eqs. (10) and (11).

Finally, it also is well known that, for a linear system, the beam's emittance is an average of the actions of individual particles:

\footnotetext{
${ }^{14}$ For example, $K_{y}(s)=\frac{e}{p_{o} c} \frac{\partial B_{y}}{\partial x}$ or $K_{x}(s)=-\frac{e}{p_{o} c} \frac{\partial B_{y}}{\partial x}+\left(\frac{e B_{y}}{p_{o} c}\right)^{2}$.
} 


$$
\varepsilon=\langle I\rangle,
$$

and averaging the second equation in (A5) over the initial betatron phase $\varphi_{o}$ shows, as expected, that emittance of the matched beam ${ }^{15}$ does not suffer from the chromaticity of the beam line:

$$
\langle I\rangle=\left\langle I_{o}\right\rangle+O\left(\delta^{2}\right)
$$

The latter is well known experimentally, viz., the chromaticity of the betatron oscillations does not result in emittance growth in a storage ring, wherein particles propagate for millions of turns and accumulate astronomical value of the phase spread due to chromaticity.

\section{APPENDIX B: EFFECTS OF FINITE BUNCH LENGTH}

Traditional TBBU treatments assume that the electron bunch is much shorter than the period of HOM oscillations. Frequently, such analysis is sufficient.

Since I am considering a possibility of a correlated energy spread, i.e., time- dependent energy chirp, it is worth considering the details of the interaction of such a beam with a dipole HOM having a frequency, $\omega_{0}$. Let us consider a bunch of electrons with rms duration $\sigma_{t}$ passing through the cavity at the HOM phase of $\varphi_{o}$. A central electron will receive a transverse kick $x_{o}^{\prime}=\theta \cdot \sin \left(\varphi_{o}\right)$. After an interval, $t$, such an electron arrives at another cavity with displacement $x=T_{12} \cdot \theta \cdot \sin \left(\varphi_{o}\right)$, and excites the EM field in the HOM with frequency, $\omega_{1}$, proportional to $A \propto e R_{\mathrm{HOM}} \cdot T_{12} \cdot \theta \cdot \sin \left(\varphi_{o}\right) \cdot \sin \left(\omega_{1} t\right)$. If the electron bunch is very short compared with the HOM oscillation period, $\omega_{o} \sigma_{t} \ll 1 ; \omega_{1} \sigma_{t} \ll 1$, all $N$ electrons will excite the EM wave coherently, and the total excited amplitude would be $A \propto q R_{\mathrm{HOM}} \cdot\left\langle T_{12}\right\rangle \cdot \theta \cdot \sin \left(\varphi_{o}\right) \cdot \sin \left(\omega_{1} t\right) ; q=$ $\mathrm{Ne}$. Otherwise, we should consider the effect of the finite bunch length on the bunch's response.

An electron deviated from the bunch center by $\tau$ would have kick $x_{o}^{\prime}=\theta \cdot \sin \left(\varphi_{o}+\omega_{o} \tau\right)$ and would excite the second cavity's HOM as follows:

$$
A \propto e R_{\mathrm{HOM}} \cdot T_{12}(\delta) \cdot \theta \cdot \sin \left(\varphi_{o}+\omega_{0} \tau\right) \cdot \sin \left[\omega_{1}(t+\tau)\right]
$$

It is useful to rewrite this in the following form:

$$
\begin{aligned}
A \propto & T_{12}(\delta) \cdot\left\{\cos \left[\varphi_{o}-\omega_{1} t+\left(\omega_{0}-\omega_{1}\right) \tau\right]\right. \\
& \left.-\cos \left[\varphi_{o}+\omega_{1} t+\left(\omega_{0}+\omega_{1}\right) \tau\right]\right\} .
\end{aligned}
$$

In the absence of a correlation between the energy deviation $\delta$ and $\tau$, and a Gaussian bunch-length distribution, $f(\tau)=\exp \left[-\left(\tau / \sigma_{t}\right)^{2} / 2\right] / \sqrt{2 \pi \sigma_{t}}$, averaging of (B2):

\footnotetext{
${ }^{15}$ Here, we assume that particles in the initial beam are distributed evenly over its betatron phases, i.e., the phase-space density is a function only of the amplitude.
}

$$
\begin{aligned}
\langle A\rangle \propto & \left\langle T_{12}\right\rangle \cdot\left\{e^{-\left(\omega_{0}-\omega_{1}\right)^{2} \sigma_{t}^{2} / 2} \cos \left(\varphi_{o}-\omega_{1} t\right)\right. \\
& \left.-e^{-\left(\omega_{0}+\omega_{1}\right)^{2} \sigma_{t}^{2} / 2} \cos \left(\varphi_{o}+\omega_{1} t\right)\right\} .
\end{aligned}
$$

While second term vanishes when $\sigma_{t} \gg 1 /\left(\omega_{1}+\omega_{o}\right)$, it is not necessarily correct for the first term, especially if $\omega_{1} \cong \omega_{o}$ :

$$
\langle A\rangle \propto\left\langle T_{12}\right\rangle \cdot\left\{\cos \left(\varphi_{o}-\omega_{0} t\right)-e^{-2 \omega_{0}^{2} \sigma_{t}^{2}} \cos \left(\varphi_{o}+\omega_{o} t\right)\right\} .
$$

In the case of correlated energy spread with $\delta=\mu \tau$; $\sigma_{\delta}=\mu \sigma_{t}$, we should use $T_{12}(\delta)=\mathrm{w}_{\mathrm{i} 0} \mathrm{w}(s, \delta) \sin \psi(s, \delta)$ and Eq. (10). Integration is trivial, and will generate four exponential terms:

$$
\exp \left[\frac{\left(\phi \pm \zeta_{1,2}\right)^{2} \sigma_{\delta}^{2}}{2}\right] ; \quad \zeta_{1,2}=\frac{\omega_{o} \pm \omega_{1}}{\mu} ;
$$

i.e., the exponential chromatic suppression will be valid when $|\phi| \gg\left|\zeta_{1,2}\right|$. In other terms, for the most interesting case of $\left|\phi \sigma_{\delta}\right| \gg 1$, the suppression projected by Eq. (16) is correct for any HOM whose period is comparable to or longer than the bunch length.

\section{APPENDIX C: ALTERNATIVE PERTURBATION METHOD}

This is a simplified version of an arbitrary case detailed in [31,32]. For a 1D linear system with Hamiltonian $\mathbf{H}=X^{T} \cdot H \cdot X ; X^{T}=\left\{x, p_{x}\right\}$, there is a full set of two eigenvectors satisfying the following equations and symplectic relations:

$$
\begin{gathered}
\tilde{Y}^{\prime}=D(s) \tilde{Y} \quad \tilde{Y}^{* \prime}=D(s) \tilde{Y}^{*} ; \quad D=\sigma \cdot H \\
\sigma=\left[\begin{array}{cc}
0 & 1 \\
-1 & 0
\end{array}\right] ; \quad \tilde{Y}=Y e^{i \psi}=\left[\begin{array}{c}
\mathrm{w} \\
(\mathrm{w}+i / \mathrm{w}) e
\end{array}\right] e^{i \psi} \\
\tilde{Y}^{* T} \sigma \tilde{Y}=Y^{* T} \sigma Y=2 i
\end{gathered}
$$

with $A^{T} \sigma A \equiv 0, \forall A$. Let us consider a slightly perturbed Hamiltonian with respect to that for an ideal case (in this case a particle with designed energy) $H=H_{o}+\delta h ; D=$ $D_{o}+\delta \Delta ;|\delta| \ll 1$. With the known eigenvectors for the ideal case:

$$
\begin{aligned}
& \tilde{Y}_{o}=\left[\begin{array}{c}
\mathrm{w}_{\mathrm{o}} \\
\left(\mathrm{w}_{\mathrm{o}}+i / \mathrm{w}_{\mathrm{o}}\right)
\end{array}\right] e^{i \psi_{\mathrm{o}}} ; \quad \tilde{Y}_{o}^{*}=\left[\begin{array}{c}
\mathrm{w}_{\mathrm{o}} e^{-i \psi_{\mathrm{o}}} \\
\left(\mathrm{w}_{\mathrm{o}}-i / \mathrm{w}_{\mathrm{o}}\right) e^{-i \psi_{\mathrm{o}}}
\end{array}\right] ; \\
& \tilde{Y}_{o}^{\prime}=D_{\mathrm{o}}(s) \tilde{Y}_{o} ; \quad \tilde{Y}_{o}^{* \prime}=D_{\mathrm{o}}(s) \tilde{Y}_{o}^{*} ; \quad D_{\mathrm{o}}(s)=\left[\begin{array}{cc}
0 & 1 \\
-K(s) & 0
\end{array}\right] .
\end{aligned}
$$

We can use perturbation theory similar to that used in quantum mechanics (see page 63 of [31]) to find perturbed solutions:

$$
\begin{gathered}
D(s)=D_{o}(s)+\delta \cdot \Delta(s) ; \quad \tilde{Y}=a \tilde{Y}_{o}+b \tilde{Y}_{o}^{*} ; \\
|b|=O(\delta) \ll|a| .
\end{gathered}
$$


Symplectic normalization $\tilde{Y}^{* T} \sigma \tilde{Y}=\left(a^{*} \tilde{Y}_{o}^{T *}+b^{*} \tilde{Y}_{o}^{T}\right) \sigma$. $\left(a \tilde{Y}_{o}+b \tilde{Y}_{o}^{*}\right)=2 i\left(|a|^{2}-|b|^{2}\right)=2 i$ imposed by $(\mathrm{C} 1)$ results in $|a|=1+O\left(\delta^{2}\right)$.

I note that even though $|a|$ does not significantly deviate from unity, the complex amplitude $a=|a| e^{i \varepsilon \chi}$ can significantly deviate from $a=1$.

Keeping only first-order terms in (C3) we derive a linear equation for $a$ and for $b$ :

$$
\begin{aligned}
\tilde{Y}^{\prime} & =\left\{D_{o}(s)+\delta \Delta(s)\right\} \tilde{Y} ; \quad \tilde{Y}_{o}^{\prime}=D_{o}(s) \tilde{Y}_{o} ; \\
a^{\prime} \tilde{Y}_{o}+b^{\prime} \tilde{Y}_{o}^{*} & =\delta \Delta(s)\left(a \tilde{Y}_{o}+b \tilde{Y}_{o}^{*}\right)=a \cdot \delta \Delta(s) \tilde{Y}_{o}+O\left(\delta^{2}\right) .
\end{aligned}
$$

Multiplying the last equation from the left by the mode projection operators $\tilde{Y}_{o}^{T *} \sigma$ :, $\tilde{Y}_{o}^{T} \sigma$ : and using an obvious $\sigma^{2}=-1$ yields the linear equations,

$$
\begin{aligned}
\tilde{Y}_{o}^{T *} \sigma: \rightarrow 2 i a^{\prime} & \cong-a \cdot \delta\left[\tilde{Y}_{o}^{T *} h(s) \tilde{Y}_{o}\right] ; \\
\tilde{Y}_{o}^{T} \sigma: \rightarrow-2 i b^{\prime} & \cong-a \cdot \delta\left[\tilde{Y}_{o}^{T} h(s) \tilde{Y}_{o}\right],
\end{aligned}
$$

that easily are solved analytically:

$$
\begin{aligned}
a & =\exp [i \delta \chi(s)] \\
\chi(s) & =\frac{1}{2} \int_{0}^{s} \tilde{Y}_{o}^{T *}(z) h(z) \tilde{Y}_{o}(z) d z ; \\
b(s) & =\frac{\delta}{2 i} \int_{0}^{s} e^{i \delta \chi(z)} \tilde{Y}_{o}^{T}(z) h(z) \tilde{Y}_{o}(z) d z .
\end{aligned}
$$

wherein I use the natural initial conditions of $a(0)=1$; $b(0)=0$. In our specific case of equation of motions for a particle with a small energy deviation

$$
h(s)=\left[\begin{array}{cc}
-K(s) & 0 \\
0 & 0
\end{array}\right]
$$

Then, Eq. (C6) becomes

$$
\begin{aligned}
a(s) & =e^{i \chi(s)} ; \quad \chi(s)=-\frac{\delta}{2} \int_{0}^{s} K(z) \mathrm{w}_{\mathrm{o}}^{2}(z) d z ; \\
b & =\frac{i \delta}{2} \int_{0}^{s} e^{i\left[2 \psi_{o}(z)+\chi(z)\right]} K(z) \mathrm{w}_{0}^{2}(z) d z .
\end{aligned}
$$

From this result it already is apparent that in a periodical lattice structure [i.e., $K(s)=K(s+C) ; Y(s+C)=Y(s)$ ], the error in the phase of the betatron oscillations, $\chi$, grows monotonically with the azimuth, while the deviation from the ideal eigenvector oscillates with double-betatron frequency plus chromatic phase shift.

Combining this with $(\mathrm{C} 2)$ we can write explicit expressions for the linear part of modification to the envelope, and the phase of the betatron motion:

$$
\mathrm{W}(\mathrm{s}) \cong \mathrm{w}_{0}(\mathrm{~s})[1+\delta \cdot v(s)] ; \quad \psi(s)=\psi_{o}(s)+\delta \cdot \phi(s) ;
$$

using straightforward manipulations:

$$
\begin{aligned}
\mathrm{w} & =\sqrt{\mathrm{w}_{\mathrm{o}}^{2}\left|a e^{i \psi_{o}(s)}+b e^{-i \psi_{o}(s)}\right|^{2}} \\
& \cong \mathrm{w}_{\mathrm{o}}^{2}\left(1+\operatorname{Re} b(s) e^{-i\left[2 \psi_{o}(s)+\chi(s)\right]}\right) \\
\psi(s) & =\operatorname{Arg}\left(a e^{i \psi_{o}(s)}+b e^{-i \psi_{o}(s)}\right) \\
& \cong \psi_{o}(s)+\chi(s)+\operatorname{Im}\left(b e^{-i\left[2 \psi_{o}(s)+\chi(s)\right]}\right) .
\end{aligned}
$$

Here, the first-order terms are identical to those derived in Appendix A with one important exception, namely, that the chromatic phase advance is involved in the envelope's modulation. While being of the same order of perturbation, the chromatic term can accumulate in a long system to a significant value and, hence, cannot be simply ignored. This paper is concerned specifically with the case wherein the accumulated chromatic phase advance can be $\gg 1$ for rms energy spread.

For completeness, I mention that for an arbitrary linear betatron coupling, the system is described completely by the full set of four four-component eigenvectors [31,32], comprised of two complex conjugated pairs $\left\{Y_{1}, Y_{1}^{*}, Y_{2}, Y_{2}^{*}\right\}, \tilde{Y}_{k}=Y_{k}(s) e^{i \psi_{k}(s)} ; k=1,2$ :

$$
\begin{gathered}
\tilde{Y}_{1,2}^{\prime}=D(s) \tilde{Y} ; \quad D=S \cdot H ; \quad S=\left[\begin{array}{cc}
\sigma & 0 \\
0 & \sigma
\end{array}\right] ; \\
\tilde{Y}_{i}^{* T} S \tilde{Y}_{k}=Y^{* T} S Y=2 i \delta_{i k} ; \quad Y_{i}^{T} S Y_{k}=0, \quad(\mathrm{C} 11)
\end{gathered}
$$

where $\delta_{i k}$ is the Kronecker's delta. For a linear perturbation, any vector can be expanded using a full set of unperturbed eigenvectors:

$$
\begin{aligned}
D(s) & =D_{o}(s)+\delta \cdot \Delta(s) ; \\
\tilde{Y}_{k} & =\left(a_{k} \tilde{Y}_{o k}+b_{k} \tilde{Y}_{o k}^{*}+c_{k} \tilde{Y}_{o i}+d_{k} \tilde{Y}_{o i}^{*}\right) ; \quad i \neq k ; \\
\left|b_{k}\right|,\left|c_{k}\right|,\left|d_{k}\right| & =O(\delta) \ll\left|a_{k}\right| \cong 1
\end{aligned}
$$

with solutions obtained employing symplectic projection operators:

$$
\begin{aligned}
\tilde{Y}_{k o}^{T *} S: \rightarrow 2 i a_{k}^{\prime} & \cong-a_{k} \cdot \delta\left[\tilde{Y}_{k o}^{T *} h(s) \tilde{Y}_{k o}\right] ; \\
\tilde{Y}_{k o}^{T} S: \rightarrow-2 i b_{k}^{\prime} & \cong \delta a_{k}\left[\tilde{Y}_{k o}^{T} h(s) \tilde{Y}_{k o}\right] ; \\
\tilde{Y}_{i o}^{T *} S: \rightarrow 2 i c_{k}^{\prime} & \cong \delta a_{k}\left[\tilde{Y}_{i o}^{T *} h(s) \tilde{Y}_{k o}\right] ; \\
\tilde{Y}_{i o}^{T} S: \rightarrow-2 i d_{k}^{\prime} & \cong \delta a_{k}\left[\tilde{Y}_{i o}^{T} h(s) \tilde{Y}_{k o}\right] ;
\end{aligned}
$$

that should be evaluated in an identical manner to that in (A7) for two pairs of $\{k, i\}:\{k=1, i=2\}$ and $\{k=2, i=1\}$. After straightforward calculations [31,32] we find that, similar to the decoupled case, the phase advance in a periodic lattice is a monotonic function of $s$ :

$$
\begin{aligned}
a_{k} & =a_{k o} e^{i \delta \chi_{k}(s)} ; \\
\chi_{k}(s) & =-\int_{0}^{s} Y_{k o}^{T *}(z) h(z) Y_{k o}(z) d z ; \quad k=1,2
\end{aligned}
$$

since in the periodic lattice the eigenvectors are periodic $Y_{k o}(z+C)=Y_{k o}(z)$ and the scalar $y=Y_{k}^{T *} h Y_{k}$ under the integral is a real number: 
1. $y^{T}=y \Rightarrow\left(Y_{k}^{T *} h Y_{k}\right)^{T}=Y_{k}^{T *} h Y_{k}$;

2. $y^{*}=\left(Y_{k}^{T^{*}} h Y_{k}\right)^{*}=Y_{k}^{T} h Y_{k}^{*}=\left(Y_{k}^{T *} h Y_{k}\right)^{T}=Y_{k}^{T *} h Y_{k}=y$,

wherein I use the fact that the Hamiltonian matrix is real $h^{*}=h$, and symmetric $h^{T}=h$.

In contrast to the traditional perturbation methods, used in Appendix A, the approach described in this section is applicable only for a linear system. However, it is not limited to the second order. ${ }^{16}$

Hence, in a nondegenerate ${ }^{17}$ periodic magnetic lattice, perturbed eigenvectors oscillate with the sum and difference betatron frequencies about the matched values, and there is no constant growth of amplitudes.

This conclusion is not always correct for the strongly mismatched case. Here, for simplicity, I consider the evolution of a strongly mismatched eigenvector for uncoupled motion (the coupled case is similar but more mathematically involved). Let us consider a properly normalized unperturbed but mismatched eigenvector:

$$
\tilde{Z}_{o}=\left(A \tilde{Y}_{o}+B \tilde{Y}_{o}^{*}\right) ; \quad|A|^{2}-|B|^{2}=1 ; \quad|B| \neq 0
$$

propagating through a periodic lattice with a matched eigenvector $Y_{o}(z+C)=Y_{o}(z)$. We easily find that $(\mathrm{C} 16)$ is the solution for unperturbed motion. The perturbation of the mismatched vector is given by Eqs. (C4)-(C8) replacing $Y \rightarrow Z$ :

$$
\begin{aligned}
\tilde{Z} & =e^{i \delta \chi(s)} \tilde{Z}_{o}+b \tilde{Z}_{o}^{*} \\
\chi(s) & =\frac{1}{2} \int_{0}^{s} \tilde{Z}_{o}^{T *}(z) h(z) \tilde{Z}_{o}(z) d z \\
b(s) & =\frac{\delta}{2 i} \int_{0}^{s} e^{i \chi(z)} \tilde{Z}_{o}^{T}(z) h(z) \tilde{Z}_{o}(z) d z
\end{aligned}
$$

Substituting (C16) in (C17) gives following expression:

$$
\begin{aligned}
\chi(s)= & \frac{1}{2} \int_{0}^{s}\left[\left(|A|^{2}+|B|^{2}\right) Y_{o}^{* T} h Y_{o}\right. \\
& \left.+2 \operatorname{Re}\left(A B^{*} \cdot Y_{o}^{T} h Y_{o} e^{2 i \psi}\right)\right] d z ; \\
b(s)= & \frac{\delta}{2 i} \int_{0}^{s} e^{i \delta \chi(z)}\left(A^{2} Y_{o}^{T} h Y_{o} e^{2 i \psi}+2 A B Y_{o}^{* T} h Y_{o}\right. \\
& \left.+B^{2} Y_{o}^{* T} h Y_{o}^{*} e^{-2 i \psi}\right) d z .
\end{aligned}
$$

and the chromaticity case (C7)

\footnotetext{
${ }^{16}$ Similar to quantum mechanics, except for some pathological and degenerate cases (such as the parametric resonance of one cell), we can extend this perturbation series to an arbitrary order as detailed in [31,32].

${ }^{17}$ The case is nondegenerate when the following is correct: $D(s+C)=D(s) ; \quad \psi_{k}(s+C)=\psi_{k}(s)+\mu_{k} ; \quad e^{2 i \mu_{k}} \neq 1 ;$ $e^{i\left(\mu_{1} \pm \mu_{2}\right)} \neq 1$.
}

$$
\begin{aligned}
\chi(s)= & -\int_{0}^{s} \frac{K(z) \mathrm{w}_{o}^{2}(z)}{2}\left[|A|^{2}+|B|^{2}\right. \\
& \left.+2 \operatorname{Re}\left(A B^{*} \cdot e^{2 i \psi(z)}\right)\right] d z ; \\
b(s)= & \frac{\delta}{2 i} \int_{0}^{s} e^{i \delta \chi(z)} K(z) \mathrm{w}_{o}^{2}(z)\left(A^{2} e^{2 i \psi}\right. \\
& \left.+2 A B+B^{2} e^{-2 i \psi}\right) d z .
\end{aligned}
$$

The second equation in (C18) significantly differs from the matched case. The second term does not oscillate and could support the steady growth of the coefficient $b$, i.e., the deviation from the unperturbed solution. In addition, there is additional phase advance for the perturbed solution $\tilde{Z}=e^{i \delta \chi(s)} \tilde{Z}_{o}+b(\delta) \tilde{Z}_{o}^{*}$; e.g., the unmatched eigenvectors spread in phase. Consequently, the projected emittance of the beam also could grow. This is a clear indication that the successful implementation of my proposed method requires properly matching the electron beam into the periodic lattice of the ERL arcs.

In other words, we can expand the initial unmatched eigenvector (describing the particle's distribution) about the perturbed matched eigenvectors:

$$
\begin{aligned}
\tilde{Z}_{o}(0) & =\left[A(\delta) \tilde{Y}(\delta)+B(\delta) \tilde{Y}^{*}(\delta)\right] ; \\
|A(\delta)|^{2}-|B(\delta)|^{2} & =1 ;
\end{aligned}
$$

at $s=0$ to find that at an arbitrary azimuth the orientation of the eigenvector

$$
\begin{aligned}
\tilde{Z}_{o}(s, \delta)= & A(\delta)\left[Y_{o}(s) e^{i[\psi(s)+\delta \chi]}+b Y_{o}^{*}(s) e^{-i \psi(s)}\right] \\
& +B(\delta)\left[Y_{o}^{*}(s) e^{-i[\psi(s)+\delta \chi]}+b^{*} Y_{o}(s) e^{i \psi(s)}\right],
\end{aligned}
$$

where the terms $b \sim O(\delta), A(\delta)-A(0) \sim O(\delta)$ stay small, while the advance of the chromatic phase is not limited. That is, in the unmatched case, the deviation of perturbed eigenvectors from the unperturbed one can be large:

$$
\begin{aligned}
& \tilde{Z}_{o}(s, \delta)-\tilde{Z}_{o}(s, 0)=A Y_{o}(s) e^{i \psi(s)}\left(e^{i \delta \chi(s)}-1\right) \\
& +B Y_{o}^{*}(s) e^{-i \psi(s)}(e-i \delta \chi(s)-1) \\
& +O(\delta) \text {. }
\end{aligned}
$$

Direct calculations, readily performed for the 1D case, show that for a mismatched beam with Gaussian energy distribution (14), the projected emittance will increase as follows:

$$
\varepsilon(s)=\varepsilon_{o} \cdot\left[1+|A B|\left(1-\mathrm{e}^{-2\left[\phi(s) \sigma_{\delta}\right]^{2}}\right)\right]+O\left(\sigma_{\delta}^{2}\right) .
$$

For the matched case $B=0$, there will be no emittance growth. For the case of interest, $\left(\phi \sigma_{\delta}\right)^{2} \gg 1$, the emittance growth is directly proportional to the mismatch: $\varepsilon(s) \cong \varepsilon_{o} \cdot(1+|A B|) .{ }^{18}$ Thus, using a chromatic lattice

\footnotetext{
${ }^{18}$ With $A=\frac{1}{2}\left(\frac{\mathrm{w}_{0}}{\mathrm{w}}+\frac{\mathrm{w}}{\mathrm{w}_{0}}\right)-\frac{i}{2}\left(\mathrm{w}_{0} \mathrm{w}^{\prime}-\mathrm{ww}_{0}^{\prime}\right) ; B=-\frac{1}{2}\left(\frac{\mathrm{w}_{0}}{\mathrm{w}}-\frac{\mathrm{w}}{\mathrm{w}_{0}}\right)+$ $\frac{i}{2}\left(\mathrm{w}_{0} \mathrm{w}^{\prime}-\mathrm{ww}_{0}^{\prime}\right)$.
} 
would require properly matching the injected beam into the ERL.

\section{APPENDIX D: CHROMATIC EFFECTS IN A GENERAL CASE}

In a generic accelerator, the energy of a particle is not a constant and could undergo both systematic changes (e.g., acceleration, deceleration, energy loss), oscillations (both synchrotron and betatron), as well as random changes (quantum fluctuations of synchrotron radiation or various scattering processes). Since longitudinal and transverse motion are fully coupled (e.g., the $K_{o} x \delta$ term in the Hamiltonian [45]), the only accurate representation of this processes is possible in a form 6D map of the complete 6D phase.

A detailed description of such a general case is very lengthy, and beyond this paper's scope. Hence, I focus here on a simple case, when the particles' longitudinal momentum is defined along the accelerator path: ${ }^{19}$

$$
p(s)=p_{o}(s)+\delta p_{o}(s)
$$

where this the energy of the reference particle. By the same reasoning, I consider a case without transverse coupling.

Since the particle's momentum no longer is a constant, we have to use canonical variables $\left\{x, P_{x}\right\},\left\{y, P_{y}\right\}$ instead of $\left\{x, x^{\prime}\right\},\left\{y, y^{\prime}\right\}$ with the Hamiltonian of

$$
\mathbf{H}=\frac{p_{x}^{2}}{2\left[p_{o}(s)+\delta p_{o}(s)\right]}+K(s) \frac{x^{2}}{2}=\mathbf{H}_{o}(s)-\nu \frac{p_{x}^{2}}{2 p_{o}(s)} .
$$

The eigenvectors of the unperturbed equation of motions have the same features and normalization as (11), but they are related to the beta function through the momentum, i.e., $\mathrm{w}^{2}=\beta / p_{o}(s)$.

The rest of the eigenvector perturbation theory described in Appendix $\mathrm{C}$ is applicable to this case:

$$
\begin{gathered}
h=-\frac{p_{x}^{2}}{2 p_{o}(s)} ; \quad \delta(s)=\frac{\delta p_{o}(s)}{p_{o}(s)} ; \\
h(s)=\left[\begin{array}{cc}
0 & 0 \\
0 & -1 / p_{o}(s)
\end{array}\right] ;
\end{gathered}
$$

with the following result:

\footnotetext{
${ }^{19}$ I note that in an accelerator the $\delta p(s)$ is not only determined by the initial momentum deviation $\delta p(0)$, but also by its arrival time at the accelerator. The latter, in turn, depends on the transverse trajectory. Only in the case of an ERL with achromatic arcs (i.e., $R_{56}=0$, etc.) is the $\delta p(s)$ fully determined by the initial momentum and time of injection, i.e., $\delta p(s)=$ $f[p(0), t(0)]$.
}

$\tilde{Y}(s)=a(s) \tilde{Y}_{o}(s)+b(s) \tilde{Y}_{o}(s) ; \quad a(s)=e^{i \chi(s)} ;$

$\chi(s)=-\frac{1}{2} \int_{0}^{s} \frac{\delta(z)}{p_{o}(z)}\left(\mathrm{w}_{o}^{\prime 2}(z)+\frac{1}{\mathrm{w}_{o}^{2}(z)}\right) d z$

$b(s)=\frac{i}{2} \int_{0}^{s} \frac{\delta(z)}{p_{o}(z)} e^{i\left[2 \psi_{o}(z)+\chi(z)\right]}\left(\mathrm{w}_{o}^{\prime}(z)+\frac{i}{\mathrm{w}_{o}(z)}\right)^{2} d z$.

One can show that for a case $\delta=$ const, $p_{o}=$ const Eq. (D5) is equivalent to (C3) and (C8). Hence, while slightly less trivial than the case of constant momentum, Eq. (D5) shows that if the sign of the momentum deviation does not change, the chromatic phase advance accumulates monotonically through the ERL lattice.

[1] M. Tigner, Nuovo Cimento 37, 1228 (1965).

[2] G. R. Neil, C. L. Bohn, S. V. Benson, G. Biallas, D. Douglas, H. F. Dylla, R. Evans, J. Fugitt, A. Grippo, J. Gubeli, R. Hill, K. Jordan, R. Li, L. Merminga, P. Piot, J. Preble, M. Shinn, T. Siggins, R. Walker, and B. Yunn, Phys. Rev. Lett. 84, 662 (2000).

[3] G.L. Carr, Michael C. Martin, Wayne R. McKinney, K. Jordan, George R. Neil, and G.P. Williams, Nature (London) 420, 153 (2002).

[4] S. M. Gruner, D. Bilderback, I. Bazarov, K. Finkelstein, G. Krafft, L. Merminga, H. Padamsee, Q. Shen, C. Sinclair, and M. Tigner, Rev. Sci. Instrum. 73, 1402 (2002).

[5] K. J. Kim, Y. Shvyd'ko, and S. Reiche, Phys. Rev. Lett. 100, 244802 (2008)

[6] I. Ben-Zvi, Ya. Derbenev, V. N. Litvinenko, and L. Merminga, Nucl. Instrum. Methods Phys. Res., Sect. A 557, 28 (2006).

[7] V. N. Litvinenko and Y. S. Derbenev, Phys. Rev. Lett. 102, 114801 (2009).

[8] V.N. Litvinenko, in The Proceedings of the 2009 Europhysics Conference on High Energy Physics (Proceeding of Science, Krakow, Poland, 2009) [http://pos .sissa.it/archive/conferences/084/141/EPS-HEP\%202009_ 141.pdf].

[9] C. M. Lyneis, M. S. McAshan, R.E. Rand, H. A. Schwettman, T.I. Smith, and J.P. Turneaure, in Proceedings of the 1979 Particle Accelerator Conference, San Francisco, CA, p. 3246, http://accelconf .web.cern.ch/AccelConf/p79/PDF/PAC1979_3246.PDF [IEEE Trans. Nucl. Sci. 26, No. 3 (1979)].

[10] P. Axel, L. S. Cardman, H. D. Graef, A. O. Hanson, R. A. Hoffswell, D. Jamnik, D.C. Sutton, R. H. Taylor, and L. M. Young, IEEE Trans. Nucl. Sci. 26, 3143 (1979).

[11] C. M. Lyneis, R.E. Rand, H. A. Schwettman, and A.M. Vetter, Nucl. Instrum. Methods 204, 269 (1983).

[12] R. E. Rand, Recirculating Electron Accelerators (Harwood Academic Publishers, New York, 1984), Sec. 9.5.

[13] R.E. Rand and T. I. Smith, Part. Accel. 11, 1 (1980) [http://cdsweb.cern.ch/record/1107981/files/p1.pdf].

[14] G. A. Krafft, J. J. Bisognano, S. Laubach, Tech-Note Report No. CEBAF-PR-90-39 [https://misportal.jlab.org/ ul/publications/view_pub.cfm?pub_id=1329]. 
[15] J. J. Bisognano and R. L. Gluckstern, in Proceedings of the 1987 Particle Accelerator Conference, Washington, DC (IEEE, Washington, DC, 1987), Catalog No. 87CH2387-9, p. 1078 .

[16] G. A. Krafft and J. J. Bisognano, in Proceedings of the 1987 Particle Accelerator Conference, Washington, DC (Ref. [15]), Catalog No. 87CH2387-9, p. 1356.

[17] G. H. Hoffstaetter and I. V. Bazarov, Phys. Rev. ST Accel. Beams 7, 054401 (2004).

[18] E. Pozdeyev, Phys. Rev. ST Accel. Beams 8, 054401 (2005).

[19] G. H. Hoffstaetter, I. V. Bazarov, and C. Song, Phys. Rev. ST Accel. Beams 10, 044401 (2007).

[20] C. D. Tennant, K. B. Beard, D. R. Kouglas, K. C. Jordan, L. Merminga, E. G. Pozdeyev, and T. Smith, Phys. Rev. ST Accel. Beams 8, 074403 (2005).

[21] H. Hahn, I. Ben-Zvi, R. Calaga, L. Hammons, V. N. Litvinenko, and W. Xu, Phys. Rev. ST Accel. Beams 13, 121002 (2010).

[22] G. Hoffstaetter (private communication), Cornell University: ERL team discusses possibility to run a test using their dedicated code for a single-pass ERL.

[23] C. W. Leemann, D. R. Douglas, and G. A. Krafft, Annu. Rev. Nucl. Part. Sci. 51, 413 (2001).

[24] V.N. Litvinenko et al., in The EIC Science Case: A Report on the Joint BNL/INT/JLab program: Gluons and the quark Sea at High Energies, edited by D. Boer, M. Diehl, R. Milner, R. Venugopal, and W. Vogelsang (Brookhaven National Laboratory, Upton, NY, 2011), p. 438 [http://arxiv.org/pdf/1108.1713v1].

[25] F. Zimmermann, F. Bordry, H.-H. Braun, O. S. Brüning, H. Burkhardt, A. de Roeck, R. Garoby, T. Linnecar, K.-H. Mess, J. Osborne, L. Rinolfi, D. Schulte, R. Tomas, J. Tuckmantel, A. Eide, F. J. Willeke, S. Chattopadhyay, B. J. Holzer, J. Dainton, M. Klein, A. Vivoli, S. Sultansoy, A. K. Ciftci, H. Aksakal, in Proceedings of the 11th European Particle Accelerator Conference, Genoa, 2008 (EPS-AG, Genoa, Italy, 2008), p. 2847.

[26] R. Calaga, I. Ben-Zvi, J. Brennan, R. Bowmann, A. Burrill, X. Chang, P. Cameron, G. Citver, D. Gassner, H. Hahn, M. Harrison, A. Hershcovitch, A. Jain, V. Litvinenko, G. McIntyre, C. Montag, A. Nicoletti, D. Kayran, A. Fedotov, J. Kewisch, W. Mackay, D. Pate, S. Peggs, J. Rank, T. Roser, J. Scaduto, T. Rao, D. Trbojevic, A. Zaltsman, K. C. Wu, Y. Zhao, H. Bluem, A. Burger, M. Cole, A. Favale, D. Holmes, J. Rathke, T. Schultheiss, and A. Todd, Nucl. Instrum. Methods Phys. Res., Sect. A 557, 243 (2006).

[27] W. Xu, I. Ben-Zvi, R. Calaga, H. Hahn, E. C. Johnson, and J. Kewisch, Nucl. Instrum. Methods Phys. Res., Sect. A 622, 17 (2010).

[28] V. Shemelin, P. Barnes, B. Gillett, M. Liepe, V. Medjidzade, H. Padamsee, R. Roy, and J. Sears, Proceedings of EPAC 2006, Edinburgh, Scotland (EPSAG, Edinburgh, Scotland, 2006), p. 468.

[29] H. Wiedeman, Particle Accelerator Physics II (SpringerVerlag, Berlin, 1995).

[30] E. E. Courant and H.S. Snyder, Ann. Phys. (N.Y.) 3, 1 (1958).

[31] V. N. Litvinenko, Selected Notes from the USPAS Winter 2008 Accelerator Physics Course [http://uspas.fnal.gov/ materials/08UCSC/Accelerator_Physics1.pdf].
[32] Vladimir N. Litvinenko, BNL Report No. C-A/AP/406, 2010 [http://www.cadops.bnl.gov/AP/ap_notes/ap_note_ 406.pdf].

[33] J. A. Crittenden, I. V. Bazarov, S. A. Belomestnykh, D. H. Bilderback, M. G. Billing, J. D. Brock, E. P. Chojnacki, B. M. Dunham, M.P. Ehrlichman, M.J. Forster, S. M. Gruner, G. H. Hoffstaetter, C. J. Johnstone, Y. Li, M. Liepe, C. E. Mayes, A. A. Mikhailichenko, H. S. Padamsee, S. B. Peck, D. C. Sagan, V. D. Shemelin, A. Temnykh, M. Tigner, and V. Veshcherevich, Proceeding of PAC09, Vancouver, BC, Canada, 2009 (IEEE, Piscataway, NJ, 2009), p. 106.

[34] T. T. Kasuga, T. Agoh, A. Enomoto, S. Fukuda, K. Furukawa, T. Furuya, K. Haga, K. Harada, S. Hiramatsu, T. Honda, K. Hosoyama, M. Izawa, E. Kako, H. Kawata, M. Kikuchi, Y. Kobayashi, M. Kuriki, T. Mitsuhashi, T. Miyajima, T. Naito, S. Nagahashi, T. Nogami, S. Noguchi, T. Obina, S. Ohsawa, M. Ono, T. Ozaki, S. Sakanaka, H. Sasaki, S. Sasaki, K. Satoh, M. Satoh, T. Shioya, T. Shishido, T. Suwada, M. Tadano, T. Takahashi, Y. Tanimoto, M. Tawada, M. Tobiyama, K. Tsuchiya, T. Uchiyama, K. Umemori, S. Yamamoto, R. Hajima, H. Iijima, N. Kikuzawa, E. J. Minehara, R. Nagai, N. Nishimori, M. Sawamura, A. Ishii, I. Ito, H. Kudoh, N. Nakamura, H. Sakai, T. Shibuya, K. Shinoe, H. Takaki, M. Katoh, A. Mochihashi, M. Shimada, H. Hanaki, and H. Tomizawa, Proceedings of PAC07, Albuquerque, New Mexico, USA, 2007 (IEEE, New York, 2007), p. 1016.

[35] T. Kasuga, T. Furuya, H. Kawata, Y. Kobayashi, S. Sakanaka, K. Satoh, N. Nakamura, and R. Hajima, Proceedings of APAC 2007, RRCAT, Indore, India (RRCAT, Indore, India, 2007), p. 172.

[36] M. Borland, G. Decker, A. Nassiri, Y. Sun, and M. White, Nucl. Instrum. Methods Phys. Res., Sect. A 582, 54 (2007).

[37] D. Trbojevic, J. Beebe-Wang, Y. Hao, V. N. Litvinenko, V. Ptitsyn, D. Kayran, and N. Tsoupas, Proceedings of Second International Particle Accelerator Conference, San Sebastian, Spain, 2011 (EPS-AG, Spain, 2011), p. 1099.

[38] D. Trbojevic, J. Beebe-Wang, Y. Hao, V. N. Litvinenko, V. Ptitsyn, D. Kayran, and N. Tsoupas, 2011 Particle Accelerator Conference, New York, NY, USA, 2011 (IEEE, New York, 2011), p. 696.

[39] G. N. Kulipanov, A. N. Skrinsky, and N. A. Vinokurov, Nucl. Instrum. Methods Phys. Res., Sect. A 467-468, 16 (2001).

[40] R. B. Neal and W. H. Panofsky, Science 152, 1353 (1966).

[41] R. H. Helm and G. A. Loew, Linear Accelerators (NorthHolland, Amsterdam, 1970).

[42] W. K. H. Panofsky and M. Bander, Rev. Sci. Instrum. 39, 206 (1968).

[43] K. A. Thompson and R. D. Ruth, Phys. Rev. D 41, 964 (1990).

[44] D. Douglas (private communication).

[45] A. A. Kolomensky and A. N. Lebedev, Theory of Cyclic Particle Accelerators (Wiley, New York, 1962).

[46] N. N. Bogolyubov and Yu. A. Mitropolsky, Asymptotic Methods in the Theory of Non-Linear Oscillations (Fizmatgiz, Moscow, 1977).

[47] Y. Hao, D. Kayran, V. N. Litvinenko, V. Ptitsyn, D. Trbojevic, and N. Tsoupas, ACC Report No. CERNLHeC-Note-2010-010, 2010 [http://cdsweb.cern.ch/ record/1323297/files/ERL\%20for\%20LHeC\%20-\%20BNL \%20paper\%20-\%20January\%202011.pdf]. 